\title{
Discontinuous generalized double-almost-periodic functions on almost-complete-closed time scales
}

\author{
Chao Wang $^{1 *}$ (D), Ravi P. Agarwal ${ }^{2,3}$, Donal O'Regan ${ }^{4}$ and Rathinasamy Sakthivel ${ }^{5}$
}

"Correspondence:

chaowang@ynu.edu.cn

'Department of Mathematics,

Yunnan University, Kunming,

Yunnan, China

Full list of author information is

available at the end of the article

\section{Springer}

\begin{abstract}
In this paper, we introduce the concept of almost-complete-closed time scales (ACCTS) that allows independent variables of functions to possess almost-periodicity under translations. For this new type of time scale, a class of piecewise functions with double-almost-periodicity is proposed and studied. Based on these, concepts of weighted pseudo-double-almost-periodic functions (WPDAP) in Banach spaces and a translation-almost-closed set are introduced. Further, we prove that the function space WPDAP 0 affiliated to WPDAP is a translation-almost-closed set. Then, by introducing the concept of almost-uniform convergence for piecewise functions on ACCTS and using measure theory on time scales, some composition theorems of WPDAP and the completeness of the function space are proved.
\end{abstract}

MSC: 26E70; 43A60

Keywords: Time scales; Double-almost-periodic functions; Weighted pseudo-double-almost-periodic; Completeness

\section{Introduction}

The theory of time scales was initiated by Hilger in 1988 [1] to unify continuous and discrete analysis, especially to combine different types of equations in hybrid domains such as $\mathbb{T}=\mathbb{R}, \mathbb{T}=q^{\mathbb{N} 0}:=\left\{q^{t}: t \in \mathbb{N}_{0}\right.$ for $\left.q>1\right\}, \mathbb{T}=\overline{q^{\mathbb{Z}}}:=q^{\mathbb{Z}} \cup\{0\}, \mathbb{T}=h \mathbb{N}$ and $\mathbb{T}=\mathbb{N}^{2}$, etc. Many works have been done in this research field (see [2-11]).

In 1927, H. Bohr developed a theory of almost-periodic functions and systems, based on this theory, almost-periodic phenomena were considered under the background of dynamic equations (see [12-15]). Since then, several different types of generalized almostperiodic functions and their related generalizations were introduced and studied and were applied to investigate the dynamical behavior of solutions with the properties of these functions (see [16-23]). Moreover, some important properties such as completeness of the function spaces and their invariance with respect to weights were established and proved (see [24-26]). Particularly, to unify the discussion of hybrid domains, the related problems of dynamic equations on time scales have been studied (see [27-31]). However, these results are based on the complete-closedness of time scales.

As is well known that most of time scales are almost-complete-closed rather than complete-closed, which will lead to the fuzziness of time scales, i.e., a time scale may

(c) The Author(s) 2019. This article is distributed under the terms of the Creative Commons Attribution 4.0 International License (http://creativecommons.org/licenses/by/4.0/), which permits unrestricted use, distribution, and reproduction in any medium, provided you give appropriate credit to the original author(s) and the source, provide a link to the Creative Commons license, and indicate if changes were made. 
approximate itself under a translation, but it will never coincide with itself. Many natural phenomena are in such a time variable structure, for instance, the time intervals of a round for a celestial body motion, the time intervals of recurrence of a tidal flood, etc. This phenomenon will cause a time approximation when almost-periodic problems are considered (see [32-34]). Hence, it is necessary to introduce and study functions on "almostcomplete-closed" time scales.

Motivated by the above, in this paper, we conduct the further discussion of the almost-complete-closed time scales, based on this, we obtain some significant properties of weighted pseudo-double-almost-periodic functions in a Banach space and establish some composition theorems.

The organization of this paper is as follows. In Sect. 2, we collect some preliminary results concerning the theory of time scales and introduce the concept of almost-completeclosed time scales (ACCTS). Also some basic properties of ACCTS are obtained and some examples are given. In Sect. 3, we introduce the concept of almost-uniform convergence of piecewise functions on ACCTS. Then we define piecewise-continuous almost-periodic functions with double periodicity. Based on this, the concepts of weighted pseudo-doublealmost-periodic functions (WPDAP) in Banach spaces and a translation-almost-closed set are introduced. Further, we prove that the function space $\mathrm{WPDAP}_{0}$ affiliated to WPDAP is a translation-almost-closed set. Then, by introducing the concept of almost-uniform convergence for piecewise functions on ACCTS and using measure theory on time scales, some composition theorems of WPDAP and the completeness of the function space are proved.

\section{Almost-complete-closed time scales (ACCTS)}

Before introducing the concept of almost-complete-closed time scales, the new concept of periodic time scales from [11] will be renamed as "complete-closed time scales" (CCTS) here.

Definition 2.1 ([11]) We say $\mathbb{T}$ is called a complete-closed time scale (CCTS) if

$$
\Pi_{0}:=\left\{\tau \in \mathbb{R}: \mathbb{T}^{\tau} \subseteq \mathbb{T}\right\} \notin\{\{0\}, \emptyset\} .
$$

We say $\Pi_{0}$ is the complete-closedness translation number set of CCTS. Furthermore, we can describe it in detail as follows:

(a) if for any $p>0$, there exists a number $P>p$ and $P \in \Pi_{0}$, we say $\mathbb{T}$ is a positive-direction CCTS;

(b) if for any $q<0$, there exists a number $Q<q$ and $Q \in \Pi_{0}$, we say $\mathbb{T}$ is a negativedirection CCTS.

(c) if $\pm \tau \in \Pi_{0}$, we say $\mathbb{T}$ is a bi-direction CCTS;

(d) we say $\mathbb{T}$ is an oriented-direction CCTS if $\mathbb{T}$ is a positive-direction CCTS or a negative-direction CCTS.

Example 2.1 Consider the following oriented-direction CCTS:

$$
\begin{array}{ll}
\mathbb{T}_{1}=\bigcup_{k=0}^{+\infty}[k(a+b), k(a+b)+a], & a, b \geq 0, a+b>0, \\
\mathbb{T}_{2}=\bigcup_{k=0}^{+\infty}[k(a+b), k(a+b)+a], & a, b \leq 0, a+b<0 .
\end{array}
$$


One can observe that $\mathbb{T}_{1}$ is a positive-direction CCTS and $\mathbb{T}_{2}$ is a negative-direction CCTS with translation number $a+b$, but they are not invariant under translations of time scales (i.e., periodic time scales) in the sense of Definition 1.1 from [10] because $\inf \mathbb{T}_{1}=$ $\sup \mathbb{T}_{2}=0$.

Remark 2.1 In fact, from [11], one can observe that CCTS can include the concept of periodic time scales which were first proposed by Kaufmann and Raffoul.

Remark 2.2 From Definition 2.1, if $\mathbb{T}$ is a complete-closed time scale, i.e., there exists some $\tau \neq 0$ such that $\mathbb{T}^{\tau} \subseteq \mathbb{T}$ (i.e., $\left(\mathbb{T}^{\tau}\right)^{-\tau} \subseteq \mathbb{T}^{-\tau}$ ), then one has $\mathbb{T} \subseteq \mathbb{T}^{-\tau}$, i.e., $\mathbb{T} \cap \mathbb{T}^{-\tau}=\mathbb{T}$, and vice versa.

According to Remark 2.2, one can obtain the following equivalent definition of CCTS immediately.

Definition 2.2 We say $\mathbb{T}$ is called a complete-closed time scale (CCTS) if

$$
\tilde{\Pi}_{0}:=\left\{\tau \in \mathbb{R}: \mathbb{T}^{-\tau} \cap \mathbb{T}=\mathbb{T}\right\} \notin\{\{0\}, \emptyset\} .
$$

We say $\tilde{\Pi}_{0}$ is the complete-closedness translation number set of CCTS.

Let $\Pi:=\left\{\tau \in \mathbb{R}: \mathbb{T}_{\tau} \neq \emptyset\right\} \neq\{0\}$, where $\mathbb{T}_{\tau}=\mathbb{T} \cap \mathbb{T}^{\tau}, \mathbb{T}^{\tau}:=\mathbb{T}+\tau=\{t+\tau: \forall t \in \mathbb{T}\}$.

In the following, we provide a lemma to guarantee that one can abstract a completeclosed time scale from an arbitrary time scale $\mathbb{T}$.

Lemma 2.1 Let $\widetilde{\Pi} \subseteq \Pi$ and $\widetilde{\Pi} \notin\{\{0\}, \emptyset\}$ be closed with respect to additive operation. If $\bigcap_{\tau \in \widetilde{\Pi}} \mathbb{T}_{\tau} \notin\{\{0\}, \emptyset\}$, then $\bigcap_{\tau \in \widetilde{\Pi}} \mathbb{T}_{\tau}$ is a complete-closed time scale.

Proof We consider the following family of sets $\mathcal{C}=\left\{\bigcap_{\tau \in A} \mathbb{T}_{\tau}: A \subset \widetilde{\Pi}\right\}$. Let $\bigcap_{\tau \in \widetilde{\Pi}} \mathbb{T}_{\tau}:=\mathbb{T}_{0}$. Obviously, $\mathbb{T}_{0} \neq \emptyset$ implies that $\mathbb{T}_{0}$ is the minimal element in the family of sets $\mathcal{C}$, and for any $\tau_{0} \in \widetilde{\Pi}$, we obtain

$$
\begin{aligned}
\mathbb{T}_{0} \cap \mathbb{T}_{0}^{\tau_{0}} & =\left(\bigcap_{\tau \in \widetilde{\Pi}} \mathbb{T}_{\tau}\right) \cap\left(\bigcap_{\tau \in \widetilde{\Pi}} \mathbb{T}_{\tau}\right)^{\tau_{0}}=\left(\bigcap_{\tau \in \widetilde{\Pi}} \mathbb{T}_{\tau}\right) \cap\left(\bigcap_{\tau \in \widetilde{\Pi}}\left(\mathbb{T}^{\tau_{0}} \cap \mathbb{T}^{\tau+\tau_{0}}\right)\right) \\
& =\left(\bigcap_{\tau \in \widetilde{\Pi}} \mathbb{T}_{\tau}\right) \cap\left(\bigcap_{\tau \in \widetilde{\Pi}} \mathbb{T}^{\tau+\tau_{0}}\right) \cap \mathbb{T}^{\tau_{0}} .
\end{aligned}
$$

Since $(\widetilde{\Pi},+)$ is closed with respect to additive operation, then $\widetilde{\Pi}+\tau_{0}:=\left\{\tau+\tau_{0}: \forall \tau \in\right.$ $\tilde{\Pi}\} \subseteq \tilde{\Pi}$. Hence, we obtain $\bigcap_{\tau \in \widetilde{\Pi}} \mathbb{T}^{\tau+\tau_{0}}=\bigcap_{\tau \in \widetilde{\Pi}+\tau_{0}} \mathbb{T}^{\tau} \supseteq \bigcap_{\tau \in \widetilde{\Pi}} \mathbb{T}^{\tau} \supset \bigcap_{\tau \in \widetilde{\Pi}} \mathbb{T}_{\tau}$. Obviously, one can also observe that $\mathbb{T}^{\tau_{0}} \supset \mathbb{T}_{\tau_{0}} \supset \bigcap_{\tau \in \tilde{\Pi}} \mathbb{T}_{\tau}$, so it follows from (2.3) that $\mathbb{T}_{0} \cap \mathbb{T}_{0}^{\tau_{0}}=$ $\bigcap_{\tau \in \widetilde{\Pi}} \mathbb{T}_{\tau}=\mathbb{T}_{0}$, which implies that $\bigcap_{\tau \in \widetilde{\Pi}} \mathbb{T}_{\tau}$ is a complete-closed time scale according to Definition 2.2. This completes the proof.

From Lemma 2.1, we obtain the following corollary directly.

Corollary 2.1 Let $\widetilde{\Pi} \subseteq \Pi$ and the pair $(\widetilde{\Pi},+)$ be an Abelian group. If $\bigcap_{\tau \in \widetilde{\Pi}} \mathbb{T}_{\tau} \notin\{\{0\}, \emptyset\}$, then $\bigcap_{\tau \in \widetilde{\Pi}} \mathbb{T}_{\tau}$ is a bi-direction CCTS. 
Example 2.2 Let $\mathbb{T}=\{-2 k, 2 k+1, k \in \mathbb{N}\} \cup\left\{\frac{2}{3}, \frac{3}{4}, \frac{n}{n+1}, n \in \mathbb{Z}^{+}\right\}$. For this time scale, we obtain $\Pi^{*}=\left\{\tilde{\Pi}_{1}, \tilde{\Pi}_{2}, \tilde{\Pi}_{2}, \tilde{\Pi}_{4}\right\} \subset \Pi$, where

$$
\begin{aligned}
& \tilde{\Pi}_{1}=\{2 k, k \in \mathbb{Z}\}, \quad \tilde{\Pi}_{2}=\left\{-\frac{2}{3},-\frac{3}{4},-\frac{n}{n+1}, n \in \mathbb{Z}^{+}\right\}, \\
& \tilde{\Pi}_{3}=\left\{2 k, k \in \mathbb{Z}^{+}\right\}, \quad \tilde{\Pi}_{4}=\left\{2 k, k \in \mathbb{Z}^{-}\right\} .
\end{aligned}
$$

We calculate that

$$
\bigcap_{\tau \in \tilde{\Pi}_{1}} \mathbb{T}_{\tau}=\left(\bigcap_{\tau \in \tilde{\Pi}_{3}} \mathbb{T}_{\tau}\right) \cap\left(\bigcap_{\tau \in \tilde{\Pi}_{4}} \mathbb{T}_{\tau}\right)=\{-2 k, k \in \mathbb{N}\} \cap\{2 k+1, k \in \mathbb{N}\}=\emptyset,
$$

and $\tilde{\Pi}_{2}$ is not closed with respect to additive operation. Hence, $\tilde{\Pi}_{1}, \tilde{\Pi}_{2}$ does not satisfy Lemma 2.1. Further,

$$
\begin{aligned}
& \mathbb{T}_{1 *}:=\bigcap_{\tau \in \tilde{\Pi}_{3}} \mathbb{T}_{\tau}=\{-2 k, k \in \mathbb{N}\} \notin\{\{0\}, \emptyset\}, \\
& \mathbb{T}_{2 *}:=\bigcap_{\tau \in \tilde{\Pi}_{4}} \mathbb{T}_{\tau}=\{2 k+1, k \in \mathbb{N}\} \notin\{\{0\}, \emptyset\},
\end{aligned}
$$

and $\tilde{\Pi}_{3}$ and $\tilde{\Pi}_{4}$ are closed with respect to additive operation, and according to Lemma 2.1, we obtain $\bigcap_{\tau \in \tilde{\Pi}_{3}} \mathbb{T}_{\tau}$ and $\bigcap_{\tau \in \tilde{\Pi}_{4}} \mathbb{T}_{\tau}$ are CCTS. In fact, through calculation, for any $\tau_{3} \in \tilde{\Pi}_{4}$ and $\tau_{4} \in \tilde{\Pi}_{3}$, one can easily obtain that $\mathbb{T}_{1 *}^{\tau_{3}} \subseteq \mathbb{T}_{1 *}$ and $\mathbb{T}_{2 *}^{\tau_{4}} \subseteq \mathbb{T}_{2 *}$.

Example 2.3 Let $\mathbb{T}=\left(\bigcup_{k=-\infty}^{+\infty}[2 k, 2 k+1]\right) \cup\left\{2 n+1+\frac{n}{n+1}, n \in \mathbb{Z}^{+}\right\} \cup\left\{2 n+\frac{n}{1-n}, n \in \mathbb{Z}^{-}\right\}$. For this time scale, we obtain $\Pi^{*}=\left\{\tilde{\Pi}_{1}, \tilde{\Pi}_{2}, \tilde{\Pi}_{3}\right\} \subset \Pi$, where

$$
\begin{aligned}
& \tilde{\Pi}_{1}=\{2 k, k \in \mathbb{Z}\}, \quad \tilde{\Pi}_{2}=\left\{-2 n-1-\frac{n}{n+1}, n \in \mathbb{Z}^{+}\right\}, \\
& \tilde{\Pi}_{3}=\left\{-2 n-\frac{n}{1-n}, n \in \mathbb{Z}^{-}\right\} .
\end{aligned}
$$

We calculate that

$$
\bigcap_{\tau \in \tilde{\Pi}_{1}} \mathbb{T}_{\tau}=\bigcup_{k=-\infty}^{+\infty}[2 k, 2 k+1] \notin\{\{0\}, \emptyset\}
$$

and $\tilde{\Pi}_{1}$ is an Abelian group. According to Corollary 2.1, $\bigcap_{\tau \in \tilde{\Pi}_{1}} \mathbb{T}_{\tau}$ is a bi-direction CCTS. In fact, through calculation, we see that $\bigcap_{\tau \in \tilde{\Pi}_{1}} \mathbb{T}_{\tau}$ is actually a bi-direction CCTS.

Let $\tau$ be a number and $A_{\tau}^{\varepsilon}$ be a subset of $\mathbb{R}, \bar{A}$ denotes the closure of the set $A$, and we set the time scales:

$$
\begin{aligned}
& \mathbb{T}:=\bigcup_{i \in I}\left[\alpha_{i}, \beta_{i}\right], \quad \mathbb{T}^{\tau}:=\mathbb{T}+\tau=\{t+\tau: \forall t \in \mathbb{T}\}:=\bigcup_{i \in I}\left[\alpha_{i}^{\tau}, \beta_{i}^{\tau}\right], \\
& \bigcup_{i \in I}\left[\tilde{\alpha}_{i}^{\tau}, \tilde{\beta}_{i}^{\tau}\right]=\overline{\mathbb{T} \backslash A_{\tau}^{\varepsilon}},
\end{aligned}
$$


and define the distance between two time scales, $\overline{\mathbb{T} \backslash A_{\tau}^{\varepsilon}}$ and $\mathbb{T}^{\tau}$, by

$$
d\left(\overline{\mathbb{T} \backslash A_{\tau}^{\varepsilon}}, \mathbb{T}^{\tau}\right)=\max \left\{\sup _{i \in I}\left|\tilde{\alpha}_{i}^{\tau}-\alpha_{i}^{\tau}\right|, \sup _{i \in I}\left|\tilde{\beta}_{i}^{\tau}-\beta_{i}^{\tau}\right|\right\}
$$

where $I$ is an infinite index set while

$$
\begin{aligned}
& \alpha_{i}^{\tau}:=\inf \left\{\alpha \in \mathbb{T}^{\tau}:\left|\alpha_{i}-\alpha\right|\right\} \quad \text { and } \quad \beta_{i}^{\tau}:=\inf \left\{\beta \in \mathbb{T}^{\tau}:\left|\beta_{i}-\beta\right|\right\} \\
& \tilde{\alpha}_{i}^{\tau}:=\inf \left\{\alpha \in \overline{\mathbb{T} \backslash A_{\tau}^{\varepsilon}}:\left|\alpha_{i}^{\tau}-\alpha\right|\right\} \quad \text { and } \quad \tilde{\beta}_{i}^{\tau}:=\inf \left\{\beta \in \overline{\mathbb{T} \backslash A_{\tau}^{\varepsilon}}:\left|\beta_{i}^{\tau}-\beta\right|\right\} .
\end{aligned}
$$

Definition 2.3 ([33]) We say that $\mathbb{T}$ is an almost-complete-closed time scale (ACCTS) if for any given $\varepsilon_{1}>0$, there exist a constant $l\left(\varepsilon_{1}\right)>0$ such that each interval of length $l\left(\varepsilon_{1}\right)$ contains a $\tau\left(\varepsilon_{1}\right)$ and sets $A_{\tau}^{\varepsilon_{1}}$ such that

$$
d\left(\overline{\mathbb{T} \backslash A_{\tau}^{\varepsilon_{1}}}, \mathbb{T}^{\tau}\right)<\varepsilon_{1}
$$

i.e., for any $\varepsilon_{1}>0$, the following set

$$
\mathrm{E}\left\{\mathbb{T}, \varepsilon_{1}\right\}=\left\{\tau \in \Pi: d\left(\overline{\mathbb{T} \backslash A_{\tau}^{\varepsilon_{1}}}, \mathbb{T}^{\tau}\right)<\varepsilon_{1}\right\}:=\Pi_{\varepsilon_{1}}
$$

is relatively dense in $\Pi$. Here, $\tau$ is called the $\varepsilon_{1}$-translation number of $\mathbb{T}, l\left(\varepsilon_{1}\right)$ is called the inclusion length of $\mathrm{E}\left\{\mathbb{T}, \varepsilon_{1}\right\}$, and $\mathrm{E}\left\{\mathbb{T}, \varepsilon_{1}\right\}$ the $\varepsilon_{1}$-translation set of $\mathbb{T}, A_{\tau}^{\varepsilon_{1}}$ is called the $\varepsilon_{1}$-improper set of $\mathbb{T}, \mathcal{R}_{\mathbb{T}}\left(\tau, \varepsilon_{1}\right):=\mathbb{T} \cap\left(\bigcup_{\tau \in \Pi_{\varepsilon_{1}}} \overline{\mathbb{T}^{-\tau} \backslash A_{-\tau}^{\varepsilon_{1}}}\right)$ the $\varepsilon_{1}$-main region of $\mathbb{T}$, where $A_{-\tau}^{\varepsilon_{1}}=\left(A_{\tau}^{\varepsilon_{1}}\right)^{-\tau}:=\left\{a-\tau: a \in A_{\tau}^{\varepsilon_{1}}\right\}$. Furthermore, we can describe it in detail as follows:

(a) if for any $p>0$, there exists a number $P>p$ and $\tau \in \mathrm{E}\left\{\mathbb{T}, \varepsilon_{1}\right\} \cap(P,+\infty)$, then we say $\mathbb{T}$ is a positive-direction ACCTS;

(b) if for any $q<0$, there exists a number $Q<q$ and $\tau \in \mathrm{E}\left\{\mathbb{T}, \varepsilon_{1}\right\} \cap(-\infty, Q)$, then we say $\mathbb{T}$ is a negative-direction ACCTS;

(c) for any $p>0, q<0$, there exist numbers $Q<q, P>p$ and $\pm \tau \in \mathrm{E}\left\{\mathbb{T}, \varepsilon_{1}\right\} \cap((-\infty, Q) \cup$ $(P,+\infty))$, then we say $\mathbb{T}$ is a bi-direction ACCTS;

(d) we say $\mathbb{T}$ is an oriented-direction ACCTS if $\mathbb{T}$ is a positive-direction ACCTS or a negative-direction ACCTS.

Remark 2.3 Definition 2.3 generalizes Definition 2.1. In fact, if we let $\varepsilon_{1} \rightarrow 0$ in Definition 2.3, then there exists $A_{\tau}^{0}=\mathbb{T} \backslash \mathbb{T}^{\tau}$ such that $d\left(\mathbb{T} \backslash A_{\tau}^{0}\right.$, $\left.\mathbb{T}^{\tau}\right)=0$, which implies that $\mathbb{T}^{\tau} \subseteq \mathbb{T}$. In fact, one can observe that if $\mathbb{T}=\mathbb{T}^{\tau}$, then the 0 -improper set $A_{\tau}^{0}=\emptyset$, i.e., $\mathbb{T}$ is a bidirection CCTS; if $\mathbb{T}^{\tau} \subset \mathbb{T}$, then $A_{\tau}^{0}=\mathbb{T} \backslash \mathbb{T}^{\tau} \neq \emptyset$, i.e., $\mathbb{T}$ is an oriented-direction CCTS.

Next, we provide some sufficient and necessary conditions to guarantee that a time scale is bi-direction CCTS or ACCTS.

Lemma 2.2 A time scale is a bi-direction CCTS if and only if there exists a 0-improper set $A_{\tau}^{0}$ such that $A_{\tau}^{0}=\emptyset$.

Proof If $\mathbb{T}$ is a bi-direction CCTS, then there exists a set $\tilde{\Pi}_{0}=\left\{\tau \in \mathbb{R}: \mathbb{T}^{\tau} \cup \mathbb{T}^{-\tau} \subseteq \mathbb{T}\right\} \neq\{0\}$, so $\mathbb{T}^{\tau} \subseteq \mathbb{T}$. For any $t \in \mathbb{T}$, we have $t-\tau \in \mathbb{T}^{-\tau} \subseteq \mathbb{T}$, thus, $t \in \mathbb{T}^{\tau}$, so we obtain $\mathbb{T} \subseteq \mathbb{T}^{\tau}$. Hence, $\mathbb{T}=\mathbb{T}^{\tau}$. From Definition 2.3, we can take the 0-improper set $A_{\tau}^{0}=\mathbb{T} \backslash \mathbb{T}^{\tau}=\emptyset$. 


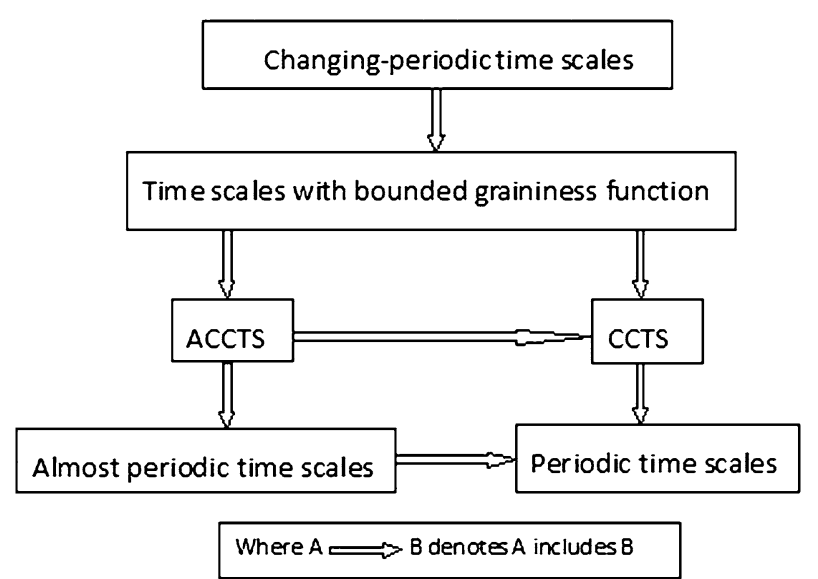

Figure 1 A new inclusion relation of time scales. New inclusion relationship among time scales

From Definition 2.3, if the 0-improper set of $\mathbb{T}$ is empty, i.e., $A_{\tau}^{0}=\emptyset$, then $d\left(\mathbb{T}, \mathbb{T}^{\tau}\right)=0$ with $\tau \neq 0$, which implies that $\mathbb{T}=\mathbb{T}^{\tau}$, so for any $t \in \mathbb{T}$, we have $t+\tau \in \mathbb{T}^{\tau}=\mathbb{T}$, i.e., $t \in \mathbb{T}^{-\tau}$, thus, $\mathbb{T} \subseteq \mathbb{T}^{-\tau}$. Furthermore, for any $t \in \mathbb{T}^{-\tau}$, we have $t+\tau \in \mathbb{T}=\mathbb{T}^{\tau}$, so $t \in \mathbb{T}$, i.e., $\mathbb{T}^{-\tau} \subseteq \mathbb{T}$. Hence, $\mathbb{T}=\mathbb{T}^{-\tau}$, i.e., $\mathbb{T}$ is a bi-direction CCTS. This completes the proof.

Lemma 2.3 A time scale is an almost-periodic time scale if and only if there exists $\varepsilon$ improper set $A_{\tau}^{\varepsilon}$ such that $\mu_{\Delta}\left(A_{\tau}^{\varepsilon}\right)<\varepsilon$.

Proof Assume that there exists the $\varepsilon$-improper set of $\mathbb{T}$ such that $\mu_{\Delta}\left(A_{\tau}^{\varepsilon}\right)<\varepsilon$, and we can take $A_{\tau}^{\varepsilon}=\mathbb{T} \backslash \mathbb{T}^{\tau}$, then it follows from $d\left(\mathbb{T} \backslash A_{\tau}^{\varepsilon}, \mathbb{T}^{\tau}\right)<\varepsilon$ that $d\left(\mathbb{T}, \mathbb{T}^{\tau}\right)=\max \left\{d\left(\mathbb{T} \backslash A_{\tau}^{\varepsilon}, \mathbb{T}^{\tau}\right)\right.$, $\left.\mu_{\Delta}\left(A_{\tau}^{\varepsilon}\right)\right\}<\varepsilon$, i.e., $\mathbb{T}$ is an almost-periodic time scale.

If $\mathbb{T}$ is an almost-periodic time scale, for any $\tau \in \Pi_{\varepsilon}$, let $A_{\tau}^{\varepsilon}=\mathbb{T} \backslash \mathbb{T}^{\tau}$, and we can obtain $\mu_{\Delta}\left(A_{\tau}^{\varepsilon}\right) \leq d\left(\mathbb{T}, \mathbb{T}^{\tau}\right)<\varepsilon$. This completes the proof.

Lemma 2.4 A time scale is an oriented-direction ACCTS if and only if there exists a $\varepsilon$ improper set $A_{\tau}^{\varepsilon}$ such that $\mu_{\Delta}\left(A_{\tau}^{\varepsilon}\right)>0$.

Proof If a time scale is ACCTS, from Definition 2.3, we can obtain that $\mu_{\Delta}\left(A_{\tau}^{\varepsilon}\right) \geq 0$. By Lemma 2.2, one can take $A_{\tau}^{0}=\emptyset$ such that $\mu_{\Delta}\left(A_{\tau}^{0}\right)=0$ if and only if $\mathbb{T}$ is bi-direction CCTS. Hence, there exists an $\varepsilon$-improper set $A_{\tau}^{\varepsilon}$ such that $\mu_{\Delta}\left(A_{\tau}^{\varepsilon}\right)>0$ if a time scale is ACCTS.

If there exists an $\varepsilon$-improper set $A_{\tau}^{\varepsilon}$ such that $\mu_{\Delta}\left(A_{\tau}^{\varepsilon}\right)>0$, according to Definition 2.3, $\mathbb{T}$ is an oriented-direction ACCTS. This completes the proof.

Remark 2.4 From the types of time scales introduced in the literature [11], we provide a new inclusion relation of time scales (see Fig. 1).

\section{Weighted piecewise pseudo-double-almost-periodic functions}

Throughout this paper, by using the same notations and definitions in Sect. 3 in [33], we will establish some fundamental results of WPDAP and $\mathrm{WPDAP}_{0}$.

In what follows, we shall assume that $\mathbb{T}$ is an almost-complete-closed time scale and denote by $\mathbb{X}$ a Banach space; let $\mathfrak{B}$ be the set consisting of all sequences $\left\{t_{k}\right\}_{k \in \mathbb{Z}}$ such that $\theta=\inf _{k \in \mathbb{Z}}\left(t_{k+1}-t_{k}\right)>0$. For $\left\{t_{k}\right\}_{k \in \mathbb{Z}} \in \mathfrak{B}$, let $\mathrm{BPC}_{r d}(\mathbb{T}, \mathbb{X})$ be the space formed by all 
bounded $r d$-piecewise continuous functions $\phi: \mathbb{T} \rightarrow \mathbb{X}$ such that $\phi(\cdot)$ is continuous at $t$ for any $t \notin\left\{t_{k}\right\}_{k \in \mathbb{Z}}$ and $\phi\left(t_{k}\right)=\phi\left(t_{k}^{-}\right)$for all $k \in \mathbb{Z}$; let $\Omega$ be a set of $\mathbb{X}$ and $\mathrm{BPC}_{r d}(\mathbb{T} \times \Omega, \mathbb{X})$ be the space formed by all bounded piecewise continuous functions $\phi: \mathbb{T} \times \Omega \rightarrow \mathbb{X}$ such that for any $x \in \Omega, \phi(\cdot, x) \in \mathrm{BPC}_{r d}(\mathbb{T}, \mathbb{X})$ and for any $t \in \mathbb{T}, \phi(t, \cdot)$ is continuous at $x \in \Omega$.

Definition 3.1 ([33]) We say $\varphi: \mathbb{T} \rightarrow \mathbb{X}$ is rd-piecewise continuous with respect to a sequence $\left\{t_{k}\right\} \subset \mathbb{T}$ which satisfy $t_{k}<t_{k+1}, k \in \mathbb{Z}$, if $\varphi(t)$ is continuous on $\left[t_{k}, t_{k+1}\right)_{\mathbb{T}}$ and rdcontinuous on $\mathbb{T} \backslash\left\{t_{k}\right\}$. Further, $\left[t_{k}, t_{k+1}\right)_{\mathbb{T}}$ are called intervals of continuity of the function $\varphi(t)$.

Similarly, one can define a class of $l d$-piecewise continuous functions. For convenience, we denote the space of all $r d$-piecewise continuous functions $\mathrm{PC}_{r d}(\mathbb{T}, \mathbb{X})$ and $\mathrm{PC}_{r d}^{\varepsilon}(\mathbb{T}, \mathbb{X}):=$ $\left\{\left.f\right|_{\mathcal{R}_{\mathbb{T}}(\tau, \varepsilon)}: f \in \mathrm{PC}_{r d}(\mathbb{T}, \mathbb{X})\right\}$.

Now, we introduce some definitions which will be used to introduce the concept of weighted piecewise pseudo-double-almost-periodic functions on ACCTS. Let $\mathfrak{B}^{\varepsilon}=$ $\left\{\left\{t_{k_{i}, \varepsilon}\right\} \subset\left\{t_{k}\right\}: t_{k_{i}} \in \mathcal{R}_{\mathbb{T}}(\tau, \varepsilon), t_{k_{i}, \varepsilon}<t_{k_{i+1}, \varepsilon}, i \in \mathbb{Z}, \lim _{i \rightarrow \infty} t_{k_{i}, \varepsilon}=\infty\right\}$.

Definition 3.2 ([33]) Let $\left\{t_{k_{i}, \varepsilon}\right\} \in \mathfrak{B}^{\varepsilon}, i \in \mathbb{Z}$. We say $\left\{t_{k_{i, \varepsilon}}^{j}\right\}$ is a $\varepsilon$-derived sequence of $\left\{t_{k_{i}, \varepsilon}\right\}$ where $t_{k_{i}, \varepsilon}^{j}=t_{k_{i+j}, \varepsilon}-t_{k_{i}, \varepsilon}, i, j \in \mathbb{Z}$.

Definition 3.3 ([33]) For any $\varepsilon_{2}>\varepsilon_{1}>0$, let $\Gamma \subset \Pi_{\varepsilon_{1}}$ be a set of real numbers and $\left\{t_{k_{i}, \varepsilon_{1}}\right\} \in \mathfrak{B}^{\varepsilon_{1}}$. We say $\left\{t_{k_{i}, \varepsilon_{1}}^{j}\right\}, i, j \in \mathbb{Z}$ is equipotentially double-almost-periodic on an almost-complete-closed time scale $\mathbb{T}$ if for $r \in \Gamma$, there exists at least one integer $q$ such that

$$
\left|t_{k_{i}, \varepsilon_{1}}^{q}-r\right|<\varepsilon_{2}, \quad \text { for all } i \in \mathbb{Z}
$$

In the following, we introduce the concept of piecewise continuous double-almostperiodic functions on ACCTS:

Definition 3.4 ([33]) Let $\mathbb{T}$ be an almost-complete-closed time scale and assume that $\left\{t_{k_{i}, \varepsilon_{1}}\right\} \in \mathfrak{B}^{\varepsilon_{1}}$ satisfying the $\varepsilon_{1}$-derived sequence $\left\{t_{k_{i}, \varepsilon_{1}}^{j}\right\}, i, j \in \mathbb{Z}$, is equipotentially almostperiodic. We call a function $\varphi \in \mathrm{PC}_{r d}^{\varepsilon_{1}}\left(\mathbb{T}, \mathbb{R}^{n}\right)$ double-almost-periodic if:

(i) for any $\varepsilon>0$, there is a positive number $\delta=\delta(\varepsilon)$ such that if the points $t^{\prime}$ and $t^{\prime \prime}$ belong to the same interval of continuity and $t^{\prime}, t^{\prime \prime} \in \mathcal{R}_{\mathbb{T}}\left(\tau, \varepsilon_{1}\right) \backslash \mathfrak{B}^{\varepsilon_{1}},\left|t^{\prime}-t^{\prime \prime}\right|<\delta$, then $\left\|\varphi\left(t^{\prime}\right)-\varphi\left(t^{\prime \prime}\right)\right\|<\varepsilon$

(ii) for any $\varepsilon_{2}>\varepsilon_{1}>0$, there is a relatively dense set $\Gamma$ of $\varepsilon_{2}$-almost-periods such that if $\tau \in \Gamma \subset \Pi_{\varepsilon_{1}}$, then $\|\varphi(t+\tau)-\varphi(t)\|<\varepsilon_{2}$ for all $t \in \mathcal{R}_{\mathbb{T}}\left(\tau, \varepsilon_{1}\right)$ which satisfy the condition $\left|t-t_{k_{i}, \varepsilon_{1}}\right|>\varepsilon_{2}, i \in \mathbb{Z}$.

We denote by $\operatorname{DAP}(\mathbb{T}, \mathbb{X})$ the space of all $r d$-piecewise double-almost-periodic func-

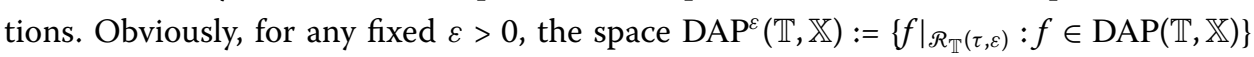
endowed with norm $\|\phi\|_{\varepsilon}=\sup _{t \in \mathcal{R}_{\mathbb{T}}(\tau, \varepsilon)}\|\phi(t)\|$ for any $\phi \in \operatorname{DAP}^{\varepsilon}(\mathbb{T}, \mathbb{X})$ is a Banach space. We also denote by $\operatorname{UPC}(\mathbb{T}, \mathbb{X})$ the space of all functions $\phi \in \mathrm{PC}_{r d}(\mathbb{T}, \mathbb{X})$ such that $\phi$ satisfies the condition (i) in Definition 3.4 and $\mathrm{UPC}^{\varepsilon}(\mathbb{T}, \mathbb{X}):=\left\{\left.f\right|_{\mathcal{R}_{\mathbb{T}}(\tau, \varepsilon)}: f \in \mathrm{UPC}(\mathbb{T}, \mathbb{X})\right\}$. Now $\mathrm{BPC}_{r d}(\mathbb{T}, \mathbb{X})$ denotes the space of all bounded $r d$-piecewise functions and $\mathrm{BPC}_{r d}^{\varepsilon}(\mathbb{T}, \mathbb{X}):=$ $\left\{\left.f\right|_{\mathcal{R}_{\mathbb{T}}(\tau, \varepsilon)}: f \in \mathrm{BPC}_{r d}(\mathbb{T}, \mathbb{X})\right\}$. 
Similarly, we can also introduce the concept of uniformly piecewise double-almostperiodic functions on almost-complete-closed time scales as follows:

Definition 3.5 ([33]) Let $\mathbb{T}$ be an almost-complete-closed time scale and assume that $\left\{t_{k_{i}, \varepsilon_{1}}\right\} \in \mathfrak{B}^{\varepsilon_{1}}$ satisfying the $\varepsilon_{1}$-derived sequence $\left\{t_{k_{i}, \varepsilon_{1}}^{j}\right\}, i, j \in \mathbb{Z}$, is equipotentially doublealmost-periodic. We call a function $f \in \mathrm{PC}_{r d}^{\varepsilon_{1}^{1}}(\mathbb{T} \times \Omega, \mathbb{X}) r d$-piecewise double-almostperiodic in $t$ uniformly in $x \in \Omega$ if:

(i) for each compact set $K \subseteq \Omega,\{f(\cdot, x): x \in K\}$ is uniformly bounded;

(ii) for any $\varepsilon>0$, there is a positive number $\delta=\delta(\varepsilon)$ such that if the points $t^{\prime}$ and $t^{\prime \prime}$ belong to the same interval of continuity and $t^{\prime}, t^{\prime \prime} \in \mathcal{R}_{\mathbb{T}}\left(\tau, \varepsilon_{1}\right) \backslash \mathfrak{B}^{\varepsilon_{1}},\left|t^{\prime}-t^{\prime \prime}\right|<\delta$, then $\left\|f\left(t^{\prime}, x\right)-f\left(t^{\prime \prime}, x\right)\right\|<\varepsilon$ for all $x \in K$;

(iii) for any $\varepsilon_{2}>\varepsilon_{1}>0$, there is relative dense set $\Gamma$ of $\varepsilon_{2}$-almost-periods such that if $\tau \in \Gamma \subset \Pi_{\varepsilon_{1}}$, then $\|f(t+\tau, x)-f(t, x)\|<\varepsilon_{2}$ for all $t \in \mathcal{R}_{\mathbb{T}}\left(\tau, \varepsilon_{1}\right), x \in K$, which satisfy the condition $\left|t-t_{k_{i}, \varepsilon_{1}}\right|>\varepsilon_{2}, i \in \mathbb{Z}$.

Now, let $U$ be the set of all functions $\rho: \mathbb{T} \rightarrow(0, \infty)$ which are positive and locally $\Delta$ integrable over $\mathbb{T}$ and let $U^{\varepsilon}:=\left\{\left.\rho\right|_{\mathcal{R}_{\mathbb{T}}(\tau, \varepsilon)}: \rho \in U\right\}$. For a given $r_{1}, r_{2} \in \mathcal{R}_{\mathbb{T}}(\tau, \varepsilon), r_{2}>r_{1}$, we set

$$
m\left(r_{1}, r_{2}, \tilde{\rho}\right):=\int_{r_{1}}^{r_{2}} \tilde{\rho}(s) \Delta s
$$

for each $\tilde{\rho} \in U^{\varepsilon}$. Let $D_{r}:=r_{2}-r_{1}$ and $\tilde{U}_{\infty}^{\varepsilon}:=\left\{\tilde{\rho} \in U^{\varepsilon}: \lim _{D_{r} \rightarrow \infty} m\left(r_{1}, r_{2}, \tilde{\rho}\right)=\infty\right\}$,

$$
\begin{aligned}
& U_{\infty}^{\varepsilon}=\left\{\tilde{\rho} \in \tilde{U}_{\infty}^{\varepsilon}: \tilde{\rho}(s) \not \equiv 0 \text { for all } s \in(t-\delta, t+\delta)_{\mathcal{R}_{\mathbb{T}}(\tau, \varepsilon)} \text {, where } t \in \mathcal{R}_{\mathbb{T}}(\tau, \varepsilon), \delta>0\right\} \\
& U_{B}^{\varepsilon}:=\left\{\tilde{\rho} \in U_{\infty}^{\varepsilon}: \tilde{\rho} \text { is bounded and } \inf _{s \in \mathcal{R}_{\mathbb{T}}(\tau, \varepsilon)} \tilde{\rho}(s)>0\right\} .
\end{aligned}
$$

It is clear that for any $\varepsilon>0, U_{B}^{\varepsilon} \subset U_{\infty}^{\varepsilon} \subset U^{\varepsilon}$. Now, for $\tilde{\rho} \in U_{\infty}^{\varepsilon}$, by (3.1), we define

$$
\operatorname{WPDAP}_{0}^{\varepsilon}(\mathbb{T}, \tilde{\rho}):=\left\{\phi \in \mathrm{BPC}_{r d}^{\varepsilon}(\mathbb{T}, \mathbb{X}): \lim _{D_{r} \rightarrow \infty} \frac{1}{m\left(r_{1}, r_{2}, \tilde{\rho}\right)} \int_{r_{1}}^{r_{2}}\|\phi(s)\| \tilde{\rho}(s) \Delta s=0\right\}
$$

Similarly, we define

$$
\begin{aligned}
& \operatorname{WPDAP}_{0}^{\varepsilon}(\mathbb{T} \times \mathbb{X}, \tilde{\rho}) \\
& \quad:=\left\{\Phi \in \mathrm{BPC}_{r d}^{\varepsilon}(\mathbb{T} \times \Omega, \mathbb{X}): \lim _{D_{r} \rightarrow \infty} \frac{1}{m\left(r_{1}, r_{2}, \tilde{\rho}\right)} \int_{r_{1}}^{r_{2}}\|\Phi(s, x)\| \tilde{\rho}(s) \Delta s=0\right.
\end{aligned}
$$

uniformly with respect to $x \in K$, where $K$ is an arbitrary compact subset of $\Omega\}$.

We are now ready to introduce the sets $\operatorname{WPDAP}^{\varepsilon}(\mathbb{T}, \tilde{\rho})$ and $\operatorname{WPDAP}^{\varepsilon}(\mathbb{T} \times \mathbb{X}, \tilde{\rho})$ of weighted pseudo-double-almost-periodic functions on ACCTS:

$$
\operatorname{WPDAP}^{\varepsilon}(\mathbb{T}, \tilde{\rho})=\left\{f=g+\phi \in \operatorname{PC}_{r d}^{\varepsilon}(\mathbb{T}, \mathbb{X}): g \in \operatorname{DAP}^{\varepsilon}(\mathbb{T}, \mathbb{X}) \text { and } \phi \in \operatorname{WPDAP}_{0}^{\varepsilon}(\mathbb{T}, \tilde{\rho})\right\} \text {, }
$$


$\operatorname{WPDAP}^{\varepsilon}(\mathbb{T} \times \mathbb{X}, \tilde{\rho})=\left\{f=g+\phi \in \mathrm{PC}_{r d}^{\varepsilon}(\mathbb{T} \times \mathbb{X}, \mathbb{X}): g \in \operatorname{DAP}^{\varepsilon}(\mathbb{T} \times \mathbb{X}, \mathbb{X})\right.$ and $\left.\phi \in \operatorname{WPDAP}_{0}^{\varepsilon}(\mathbb{T} \times \mathbb{X}, \tilde{\rho})\right\}$.

Theorem 3.1 For any $r \in \Pi_{\varepsilon}$ and a set $\left\{t_{n}\right\}:=\Lambda^{*} \subset\left[t_{0}-r, t_{0}+r\right)_{\mathbb{T}}$, where $t_{0} \in \mathbb{T}$, there exists $C_{1}\left(\Lambda^{*}\right), C_{2}\left(\Lambda^{*}\right)>0$ such that

$$
C_{1}\left(\Lambda^{*}\right) \sum_{t_{j} \in\left[t_{0}-r, t_{0}+r\right)_{\mathbb{T}}} \underline{\tilde{\rho}}\left(t_{j}\right) \leq \sum_{t_{j} \in\left[t_{0}-r, t_{0}+r\right)_{\mathbb{T}}} \overline{\tilde{\rho}}\left(t_{j}\right) \leq C_{2}\left(\Lambda^{*}\right) \sum_{t_{j} \in\left[t_{0}-r, t_{0}+r\right)_{\mathbb{T}}} \underline{\tilde{\rho}}\left(t_{j}\right),
$$

where $\underline{\tilde{\rho}}\left(t_{j}\right)=\inf \left\{\tilde{\rho}(t): t \in\left[t_{j}, t_{j+1}\right)_{\mathbb{T}}\right\}$ and $\overline{\tilde{\rho}}\left(t_{j}\right)=\sup \left\{\tilde{\rho}(t): t \in\left[t_{j}, t_{j+1}\right)_{\mathbb{T}}\right\}$.

Proof Since $\tilde{\rho}: \mathbb{T} \rightarrow(0, \infty)$ is locally $\Delta$-integrable over $\mathbb{T}$, then there exists a partition $\Lambda^{*}$ as follows

$$
t_{0}-r:=t_{1}<t_{2}<\cdots<t_{n-1}<t_{n}:=t_{0}+r
$$

such that

$$
\left(\sum_{t_{j} \in\left[t_{0}-r, t_{0}+r\right)_{\mathbb{T}}} \overline{\tilde{\rho}}\left(t_{j}\right)-\sum_{t_{j} \in\left[t_{0}-r, t_{0}+r\right)_{\mathbb{T}}} \underline{\tilde{\rho}}\left(t_{j}\right)\right)\left(t_{j+1}-t_{j}\right)<\varepsilon\left(\Lambda^{*}\right) .
$$

Denote $\delta\left(\Lambda^{*}\right):=\inf \left(t_{j+1}-t_{j}\right)$, then

$$
\sum_{t_{j} \in\left[t_{0}-r, t_{0}+r\right)_{\mathbb{T}}} \overline{\tilde{\rho}}\left(t_{j}\right)-\sum_{t_{j} \in\left[t_{0}-r, t_{0}+r\right)_{\mathbb{T}}} \tilde{\rho}\left(t_{j}\right)<\frac{\varepsilon\left(\Lambda^{*}\right)}{\delta\left(\Lambda^{*}\right)},
$$

which implies that

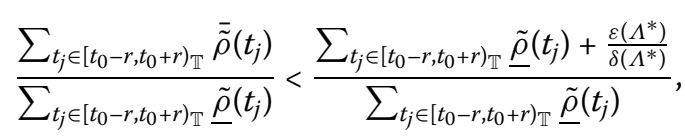

so, obviously, we have

$$
C_{1}\left(\Lambda^{*}\right)=1 \leq \frac{\sum_{t_{j} \in\left[t_{0}-r, t_{0}+r\right)_{\mathbb{T}}} \overline{\tilde{\rho}}\left(t_{j}\right)}{\sum_{t_{j} \in\left[t_{0}-r, t_{0}+r\right)_{\mathbb{T}}} \tilde{\tilde{\rho}}\left(t_{j}\right)} \leq 1+\frac{\frac{\varepsilon\left(\Lambda^{*}\right)}{\delta\left(\Lambda^{*}\right)}}{\sum_{t_{j} \in\left[t_{0}-r, t_{0}+r\right)_{\mathbb{T}}} \underline{\tilde{\rho}}\left(t_{j}\right)}=C_{2}\left(\Lambda^{*}\right) .
$$

This completes the proof.

Definition 3.6 In Theorem 3.1, the finite set $\Lambda^{*}$ is said to be a discretization partition of $m\left(r, \tilde{\rho}, t_{0}\right)$ and $\sum_{t_{j} \in\left[t_{0}-r, t_{0}+r\right)_{\mathbb{T}}} \underline{\tilde{\rho}}\left(t_{j}\right)$ and $\sum_{\left.t_{j} \in\left[t_{0}-r, t_{0}+r\right)\right]_{\mathbb{T}}} \overline{\tilde{\rho}}\left(t_{j}\right)$ are called the equivalent discrete weights under the discretization partition $\Lambda^{*}$.

Lemma 3.1 Let $\phi \in \mathrm{BPC}_{r d}^{\varepsilon_{1}}(\mathbb{T}, \mathbb{X})$. Then, $\phi \in \operatorname{WPDAP}_{0}^{\varepsilon_{1}}(\mathbb{T}, \tilde{\rho})$ where $\tilde{\rho} \in U_{\infty}^{\varepsilon_{1}}$ if and only if for every $\varepsilon_{2}>\varepsilon_{1}>0$,

$$
\lim _{D_{r} \rightarrow \infty} \frac{1}{m\left(r_{1}, r_{2}, \tilde{\rho}\right)} \int_{M_{r_{1}, r_{2}, \varepsilon_{2}}(\phi)} \tilde{\rho}(t) \Delta t=0,
$$

and $M_{r_{1}, r_{2}, \varepsilon_{2}}(\phi):=\left\{t \in\left[r_{1}, r_{2}\right]_{\mathcal{R}_{\mathbb{T}}\left(\tau, \varepsilon_{1}\right)}:\|\phi(t)\| \geq \varepsilon_{2}\right\}$. 
Proof (a) Necessity. For contradiction, suppose that there exist $\varepsilon_{2}^{0}>\varepsilon_{1}^{0}>0$ such that

$$
\lim _{D_{r} \rightarrow \infty} \frac{1}{m\left(r_{1}, r_{2}, \tilde{\rho}\right)} \int_{M_{r_{1}, r_{2}, \varepsilon_{2}^{0}}(\phi)} \tilde{\rho}(t) \Delta t \neq 0 .
$$

Then, there exists $\delta>0$ such that

$$
\frac{1}{m\left(r_{1}^{n_{1}}, r_{2}^{n_{2}}, \tilde{\rho}\right)} \int_{M_{r_{1}^{n_{1}, r_{2}, \varepsilon_{2}} n_{2}(\phi)}} \tilde{\rho}(t) \Delta t \geq \delta,
$$

where $r_{1}^{n_{1}} \leq\left\lceil r_{n_{1}}\right\rceil:=n_{1}, r_{2}^{n_{2}} \geq\left\lfloor r_{2}^{n_{2}}\right\rfloor:=n_{2}$ and $r_{1}^{n_{1}} \leq r_{2}^{n_{2}}, r_{1}^{n_{1}}, r_{2}^{n_{2}} \in \mathcal{R}_{\mathbb{T}}\left(\tau, \varepsilon_{1}^{0}\right)$.

Thus, we have

$$
\begin{aligned}
& \frac{1}{m\left(r_{1}^{n_{1}}, r_{2}^{n_{2}}, \tilde{\rho}\right)} \int_{r_{1}^{n_{1}}}^{r_{2}^{n_{2}}}\|\phi(s)\| \tilde{\rho}(s) \Delta s \\
& =\frac{1}{m\left(r_{1}^{n_{1}}, r_{2}^{n_{2}}, \tilde{\rho}\right)} \int_{M_{r_{1} n_{1}, r_{2}, \varepsilon_{2}}^{n_{2}}(\phi)}\|\phi(s)\| \tilde{\rho}(s) \Delta s
\end{aligned}
$$

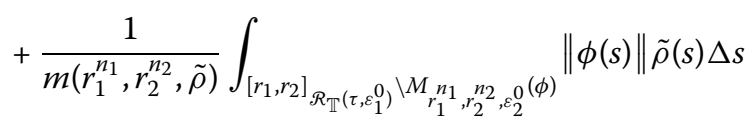

$$
\begin{aligned}
& \geq \frac{1}{m\left(r_{1}^{n_{1}}, r_{2}^{n_{2}}, \tilde{\rho}\right)} \int_{M_{r_{1}^{n_{1}}, r_{2}^{n_{2}, \varepsilon_{2}^{(0)}}}(\phi)}\|\phi(s)\| \tilde{\rho}(s) \Delta s \\
& \geq \frac{\varepsilon_{2}^{0}}{m\left(r_{1}^{n_{1}}, r_{2}^{n_{2}}, \tilde{\rho}\right)} \int_{M_{r_{1}^{n_{1}, r_{2}, r_{2}} \varepsilon_{2}^{(\phi)}}} \tilde{\rho}(s) \Delta s \geq \varepsilon_{2}^{0} \delta,
\end{aligned}
$$

and this contradicts the assumption.

(b) Sufficiency. Let $M:=\sup _{t \in \mathcal{R}_{\mathbb{T}}\left(\tau, \varepsilon_{1}\right)}\|\phi(t)\|<\infty$. Assume that

$$
\lim _{D_{r} \rightarrow \infty} \frac{1}{m\left(r_{1}, r_{2}, \tilde{\rho}\right)} \int_{M_{r_{1}, r_{2}, \varepsilon_{2}}(\phi)} \tilde{\rho}(t) \Delta t=0 .
$$

Then, for every $\varepsilon_{2}>\varepsilon_{1}>0$, there exists $r_{0}>0$ such that for every $D_{r}>r_{0}$,

$$
\frac{1}{m\left(r_{1}, r_{2}, \tilde{\rho}\right)} \int_{M_{r_{1}, r_{2}, \varepsilon_{2}(\phi)}} \tilde{\rho}(t) \Delta t<\frac{\varepsilon_{2}}{M}
$$

Now, we have

$$
\begin{aligned}
& \frac{1}{m\left(r_{1}, r_{2}, \tilde{\rho}\right)} \int_{r_{1}}^{r_{2}}\|\phi(s)\| \tilde{\rho}(s) \Delta s \\
& =\frac{1}{m\left(r_{1}, r_{2}, \tilde{\rho}\right)}\left(\int_{M_{r_{1}, r_{2}, \varepsilon_{2}}(\phi)}\|\phi(s)\| \tilde{\rho}(s) \Delta s\right. \\
& \left.+\int_{\left[r_{1}, r_{2}\right]_{\mathbb{R}_{\mathbb{T}}\left(\tau, \varepsilon_{1}\right)} \backslash M_{r_{1}, r_{2}, \varepsilon_{2}}(\phi)}\|\phi(s)\| \tilde{\rho}(s) \Delta s\right) \\
& \leq \frac{M}{m\left(r_{1}, r_{2}, \tilde{\rho}\right)} \int_{M_{r_{1}, r_{2}, \varepsilon_{2}}(\phi)} \tilde{\rho}(t) \Delta t \\
& +\frac{\varepsilon_{2}}{m\left(r_{1}, r_{2}, \tilde{\rho}\right)} \int_{\left[r_{1}, r_{2}\right]_{\mathbb{R}_{\mathbb{T}}\left(\tau, \varepsilon_{1}\right)} \backslash M_{r_{1}, r_{2}, \varepsilon_{2}}(\phi)} \tilde{\rho}(s) \Delta s \leq 2 \varepsilon_{2} .
\end{aligned}
$$


Therefore, it follows that

$$
\lim _{D_{r} \rightarrow \infty} \frac{1}{m\left(r_{1}, r_{2}, \tilde{\rho}\right)} \int_{\left[r_{1}, r_{2}\right]_{\mathcal{R}_{\mathbb{T}}\left(\tau, \varepsilon_{1}\right)}}\|\phi(s)\| \tilde{\rho}(s) \Delta s=0
$$

that is, $\phi \in \operatorname{WPDAP}_{0}^{\varepsilon_{1}}(\mathbb{T}, \tilde{\rho})$. This completes the proof.

From the proof of Lemma 3.1, the following corollary is immediate.

Corollary 3.1 Let $\phi \in \mathrm{BPC}_{r d}^{\varepsilon_{1}}(\mathbb{T}, \mathbb{X})$. Then, $\phi \in \operatorname{WPDAP}_{0}^{\varepsilon_{1}}(\mathbb{T}, \tilde{\rho})$ where $\rho \in U_{\infty}^{\varepsilon_{1}}$ if and only iffor every $\varepsilon_{2}>\varepsilon_{1}>0$,

$$
\lim _{D_{r} \rightarrow \infty} \frac{1}{m\left(r_{1}, r_{2}, \tilde{\rho}\right)} \mu_{\Delta}\left(M_{r_{1}, r_{2}, \varepsilon_{2}}(\phi)\right)=0
$$

and $M_{r_{1}, r_{2}, \varepsilon_{2}}(\phi):=\left\{t \in\left[r_{1}, r_{2}\right]_{\mathcal{R}_{\mathbb{T}}\left(\tau, \varepsilon_{1}\right)}:\|\phi(t)\| \geq \varepsilon_{2}\right\}$

Lemma 3.2 Let $\mathbb{T}$ be an almost-complete-closed time scale. Then, $\operatorname{WPDAP}_{0}^{\varepsilon}(\mathbb{T}, \tilde{\rho})$ is a translation-almost-closed set of $\mathrm{BPC}_{r d}^{\varepsilon}(\mathbb{T}, \mathbb{X})$ if $\tilde{\rho} \in U_{\infty}^{\varepsilon}$, i.e., $\phi(t+s):=\theta_{s} \phi \in \operatorname{WPDAP}_{0}^{\varepsilon}(\mathbb{T}, \tilde{\rho})$, $t \in \mathcal{R}_{\mathbb{T}}(\tau, \varepsilon), s \in \Pi_{\varepsilon}$, if $\tilde{\rho} \in U_{\infty}^{\varepsilon}$.

Proof For $\phi \in \operatorname{WPDAP}_{0}^{\varepsilon_{1}}(\mathbb{T}, \tilde{\rho}), \varepsilon_{2}>\varepsilon_{1}>0, r_{1}, r_{2} \in \mathcal{R}_{\mathbb{T}}\left(\tau, \varepsilon_{1}\right)$, we have

$$
\begin{aligned}
M_{r_{1}, r_{2}, \varepsilon_{2}}\left(\theta_{s} \phi\right) & =\left\{t \in\left[r_{1}, r_{2}\right]_{\mathcal{R}_{\mathbb{T}}\left(\tau, \varepsilon_{1}\right)}:\left\|\theta_{s}(t)\right\| \geq \varepsilon_{2}\right\} \\
& =\left\{t \in\left[r_{1}, r_{2}\right]_{\mathcal{R}_{\mathbb{T}}\left(\tau, \varepsilon_{1}\right)}:\|\phi(t+s)\| \geq \varepsilon_{2}\right\} \\
& =\left\{t \in\left[r_{1}+s, r_{2}+s\right]_{\mathcal{R}_{\mathbb{T}}\left(\tau, \varepsilon_{1}\right)}:\|\phi(t)\| \geq \varepsilon_{2}\right\} \\
& \subseteq\left\{t \in\left[\tilde{r}_{1}, \tilde{r}_{2}\right]_{\mathcal{R}_{\mathbb{T}}\left(\tau, \varepsilon_{1}\right)}:\|\phi(t)\| \geq \varepsilon_{2}\right\},
\end{aligned}
$$

where $\tilde{r}_{1}, \tilde{r}_{2} \in \mathcal{R}_{\mathbb{T}}\left(\tau, \varepsilon_{1}\right)$ and $\tilde{r}_{1}<r_{1}+s$ and $\tilde{r}_{2}>r_{2}+s$. Let $D_{\tilde{r}}:=\tilde{r}_{2}-\tilde{r}_{1}$, so $D_{r} \rightarrow+\infty$ implies $D_{\tilde{r}} \rightarrow+\infty$.

Hence, it follows that

$$
\begin{aligned}
\frac{1}{m\left(r_{1}, r_{2}, \tilde{\rho}\right)} \mu_{\Delta}\left(M_{r_{1}, r_{2}, \varepsilon_{2}}\left(\theta_{s} \phi\right)\right) & \leq \frac{1}{m\left(r_{1}, r_{2}, \tilde{\rho}\right)} \mu_{\Delta}\left(M_{\tilde{r}_{1}, \tilde{r}_{2}, \varepsilon_{2}}\left(\theta_{s} \phi\right)\right) \\
& =\frac{m\left(\tilde{r}_{1}, \tilde{r}_{2}, \tilde{\rho}\right)}{m\left(r_{1}, r_{2}, \tilde{\rho}\right)} \frac{1}{m\left(\tilde{r}_{1}, \tilde{r}_{2}, \tilde{\rho}\right)} \mu_{\Delta}\left(M_{r_{1}, r_{2}, \varepsilon_{2}}(\phi)\right) .
\end{aligned}
$$

Since $\phi \in \operatorname{WPDAP}_{0}^{\varepsilon_{1}}(\mathbb{T}, \tilde{\rho})$, from Lemma 3.1, we have

$$
\frac{1}{m\left(\tilde{r}_{1}, \tilde{r}_{2}, \tilde{\rho}\right)} \mu_{\Delta}\left(M_{\tilde{r}_{1}, \tilde{r}_{2}, \varepsilon_{2}}(\phi)\right) \rightarrow 0, \quad D_{r} \rightarrow \infty .
$$

Further, $\lim _{D_{r} \rightarrow \infty} \frac{m\left(\tilde{r}_{1}, \tilde{r}_{2}, \tilde{\rho}\right)}{m\left(r_{1}, r_{2}, \tilde{\rho}\right)}=1$, and thus

$$
\frac{1}{m\left(r_{1}, r_{2}, \tilde{\rho}\right)} \mu_{\Delta}\left(M_{r_{1}, r_{2}, \varepsilon_{2}}\left(\theta_{s}(\phi)\right)\right) \rightarrow 0, \quad D_{r} \rightarrow \infty
$$

Again, using Lemma 3.1, one finds $\theta_{s} \phi \in \operatorname{WPDAP}_{0}^{\varepsilon 1}(\mathbb{T}, \tilde{\rho})$. This completes the proof. 
Let $T, P \in \mathfrak{B}^{\varepsilon}$ and let $s_{\varepsilon}(T \cup P): \mathfrak{B}^{\varepsilon} \rightarrow \mathfrak{B}^{\varepsilon}$ be a map such that the set $s_{\varepsilon}(T \cup P)$ forms a strictly increasing sequence for any fixed $\varepsilon>0$. Let $D \subset \mathcal{R}_{\mathbb{T}}(\tau, \varepsilon)$, and we introduce the notations $D^{\xi}=\{t+\xi: t \in D\}, F_{\xi}(D)=D \cap D^{\xi}$. We denote by $\tilde{\phi}=(\varphi(t), T)$ the element from the space $\mathrm{PC}_{r d}^{\varepsilon}(\mathbb{T}, \mathbb{X}) \times \mathfrak{B}^{\varepsilon}$, and for every sequence of real numbers $\left\{s_{n}\right\}, n=1,2, \ldots$ with $\theta_{s_{n}} \tilde{\phi}$, we consider the sets $\left\{\varphi\left(t+s_{n}\right), T^{-s_{n}}\right\} \subset \mathrm{PC}_{r d}^{\varepsilon} \times \mathfrak{B}^{\varepsilon}$, where $T^{-s_{n}}=\left\{t_{k_{i}}-s_{n}: i \in \mathbb{Z}, n=\right.$ $1,2, \ldots\}$.

Next, we introduce the convergent form of piecewise functions on almost-completeclosed time scales:

Definition 3.7 (Almost-uniform convergence for piecewise functions) Let $\mathbb{T}$ be an almost-complete-closed time scale. The sequence $\left\{\tilde{\phi}_{n}\right\}, \tilde{\phi}_{n}=\left(\varphi_{n}(t), T_{n}\right) \in \mathrm{PC}_{r d}^{\varepsilon_{1}}(\mathbb{T}, \mathbb{X}) \times \mathfrak{B}^{\varepsilon_{1}}$ is almost-convergent to $\tilde{\phi}, \tilde{\phi}=(\varphi(t), T),(\varphi(t), T) \in \mathrm{PC}_{r d}^{\varepsilon_{1}}(\mathbb{T}, \mathbb{X}) \times \mathfrak{B}^{\varepsilon_{1}}$ if and only if for any $\varepsilon_{2}>\varepsilon_{1}>0$ there exists $n_{0}>0$ such that $n \geq n_{0}$ implies

$$
\tilde{d}\left(T, T_{n}\right)<\varepsilon_{2}, \quad\left\|\varphi_{n}(t)-\varphi(t)\right\|<\varepsilon_{2}
$$

uniformly for $t \in \mathcal{R}_{\mathbb{T}}\left(\tau, \varepsilon_{1}\right) \backslash F_{\varepsilon_{2}}\left(s_{\varepsilon_{1}}\left(T_{n} \cup T\right)\right)$, here $\tilde{d}(\cdot, \cdot)$ is an arbitrary distance in $\mathfrak{B}^{\varepsilon_{1}}$.

Remark 3.1 The convergence described by Definition 3.7 is a distinct convergence form on ACCTS, which will contribute to studying approximation of functions under the time scale that is almost the same as each other under translations. Traditionally, researchers analyze functions on complete-closed time scales, which provides no difficulties in introducing and studying functions, especially for some important and basic functions like almostperiodic functions, almost-automorphic functions, and so on, because such functions are defined by their translations on the domain and their domain is at least a complete-closed time scale. However, in the Introduction part of this paper and recent works [32-34], ACCTS are a class of necessary and important time variable forms that exist. Obviously, if we let $\varepsilon_{1} \rightarrow 0$ in Definition 3.7, then one can easily obtain the convergence form for piecewise functions on CCTS.

In view of Definition 3.7, we now introduce the second definition of piecewise continuous double-almost-periodic functions on ACCTS:

Definition 3.8 Let $\mathbb{T}$ be an almost-complete-closed time scale. The function $\varphi \in \mathrm{PC}_{r d}^{\varepsilon}(\mathbb{T}$, $\mathbb{X})$ is said to be $r d$-piecewise continuous double-almost-periodic with respect to a sequence from the set $T \in \mathfrak{B}^{\varepsilon}$ if for every sequence of real numbers $\left\{s_{m}^{\prime}\right\} \subset \Pi_{\varepsilon}$ there exists a subsequence $\left\{s_{n}\right\}, s_{n}=s_{m_{n}}^{\prime}$ and a sequence $\left\{A_{-s_{n}}\right\}$ such that the limit set $\mathbb{T}_{0}$ of $\left\{\mathbb{T}^{-s_{n}} \backslash A_{-s_{n}}\right\}$ exists and $\theta_{s_{n}} \tilde{\phi}$ is uniformly convergent on $\mathrm{PC}_{r d}\left(\mathbb{T}_{0}, \mathbb{X}\right) \times \mathfrak{B}_{0}$, where $\mathfrak{B}_{0}:=\mathbb{T}_{0} \cap \mathfrak{B}$.

From Definition 3.8, two lemmas below are immediate:

Lemma 3.3 Let $\phi \in \operatorname{DAP}^{\varepsilon}(\mathbb{T}, \mathbb{X})$, then for any $\varepsilon>0$, the almost-range of $\phi, \phi\left(\mathcal{R}_{\mathbb{T}}(\tau, \varepsilon)\right)$, is a relatively compact subset of $\mathbb{X}$.

Lemma 3.4 If $f=g+\phi$ with $g \in \operatorname{DAP}^{\varepsilon}(\mathbb{T}, \mathbb{X})$, and $\phi \in \operatorname{WPDAP}_{0}^{\varepsilon}(\mathbb{T}, \rho)$, where $\tilde{\rho} \in U_{\infty}^{\varepsilon}$, then $g\left(\mathcal{R}_{\mathbb{T}}(\tau, \varepsilon)\right) \subset \overline{f\left(\mathcal{R}_{\mathbb{T}}(\tau, \varepsilon)\right)}$. 
Lemma 3.5 For any $\varepsilon>0$, one can decompose a weighted piecewise pseudo-double-almostperiodic function according to $\mathrm{DAP}^{\varepsilon} \oplus \operatorname{WPDAP}_{0}^{\varepsilon}$ which is unique for any $\tilde{\rho} \in U_{\infty}^{\varepsilon}$.

Proof Assume that $f=g_{1}+\phi_{1}$ and $f=g_{2}+\phi_{2}$. Then, $\left(g_{1}-g_{2}\right)+\left(\phi_{1}-\phi_{2}\right)=0$. Since $g_{1}-$ $g_{2} \in \operatorname{DAP}^{\varepsilon}(\mathbb{T}, \mathbb{X})$, and $\phi_{1}-\phi_{2} \in \operatorname{WPDAP}_{0}^{\varepsilon}(\mathbb{T}, \tilde{\rho})$, we find that $g_{1}-g_{2}=0$. Consequently, $\phi_{1}-\phi_{2}=0$, i.e., $\phi_{1}=\phi_{2}$. This completes the proof.

Theorem 3.2 For $\tilde{\rho} \in U_{\infty}^{\varepsilon},\left(\operatorname{WPDAP}^{\varepsilon}(\mathbb{T}, \tilde{\rho}),\|\cdot\|_{\varepsilon}\right)$ is a Banach space for any $\varepsilon>0$.

Proof Assume that $\left\{f_{n}\right\}_{n \in \mathbb{N}}$ is a Cauchy sequence in $\operatorname{WPDAP}^{\varepsilon}(\mathbb{T}, \tilde{\rho})$. We can write $f_{n}=g_{n}+$ $\phi_{n}$ uniquely. Using Lemma 3.4, we have $\left\|g_{p}-g_{q}\right\|_{\varepsilon} \leq\left\|f_{p}-f_{q}\right\|_{\varepsilon}$, from which we deduce that $\left\{g_{n}\right\}_{n \in \mathbb{N}}$ is a Cauchy sequence in $\operatorname{DAP}^{\varepsilon}(\mathbb{T}, \mathbb{X})$. Hence, $\phi_{n}=f_{n}-g_{n}$ is a Cauchy sequence in $\operatorname{WPDAP}_{0}^{\varepsilon}(\mathbb{T}, \tilde{\rho})$. Thus, $g_{n} \rightarrow g \in \operatorname{DAP}^{\varepsilon}(\mathbb{T}, \mathbb{X}), \phi_{n} \rightarrow \phi \in \operatorname{WPDAP}_{0}^{\varepsilon}(\mathbb{T}, \tilde{\rho})$, and finally, $f_{n} \rightarrow$ $g+\phi \in \operatorname{WPDAP}^{\varepsilon}(\mathbb{T}, \tilde{\rho})$. This completes the proof.

Definition 3.9 Let $\tilde{\rho}_{1}, \tilde{\rho}_{2} \in U_{\infty}^{\varepsilon}$. We say that $\tilde{\rho}_{1}$ is $\varepsilon$-equivalent to $\tilde{\rho}_{2}$, written as $\tilde{\rho}_{1} \sim^{\varepsilon} \tilde{\rho}_{2}$, if $\tilde{\rho}_{1} / \tilde{\rho}_{2} \in U_{B}^{\varepsilon}$.

Theorem 3.3 Let $\tilde{\rho}_{1}, \tilde{\rho}_{2} \in U_{\infty}^{\varepsilon}$. If $\tilde{\rho}_{1} \sim^{\varepsilon} \tilde{\rho}_{2}$, then $\operatorname{WPDAP}^{\varepsilon}\left(\mathbb{T}, \tilde{\rho}_{1}\right)=\operatorname{WPDAP}^{\varepsilon}\left(\mathbb{T}, \tilde{\rho}_{2}\right)$.

Proof Assume that $\tilde{\rho}_{1} \sim^{\varepsilon} \tilde{\rho}_{2}$. Then, there exist $a, b>0$ such that $a \tilde{\rho}_{1} \leq \tilde{\rho}_{2} \leq b \tilde{\rho}_{1}$. Thus, for any $r_{1}, r_{2} \in \mathcal{R}_{\mathbb{T}}(\tau, \varepsilon)$, we have $a m\left(r_{1}, r_{2}, \tilde{\rho}_{1}\right) \leq m\left(r_{1}, r_{2}, \tilde{\rho}_{2}\right) \leq b m\left(r_{1}, r_{2}, \tilde{\rho}_{1}\right)$, and

$$
\begin{aligned}
\frac{a}{b} \frac{1}{m\left(r_{1}, r_{2}, \tilde{\rho}_{1}\right)} \int_{r_{1}}^{r_{2}}\|\phi(s)\| \tilde{\rho}_{1}(s) \Delta s & \leq \frac{1}{m\left(r_{1}, r_{2}, \tilde{\rho}_{2}\right)} \int_{r_{1}}^{r_{2}}\|\phi(s)\| \tilde{\rho}_{2}(s) \Delta s \\
& \leq \frac{b}{a} \frac{1}{m\left(r_{1}, r_{2}, \tilde{\rho}_{1}\right)} \int_{r_{1}}^{r_{2}}\|\phi(s)\| \tilde{\rho}_{1}(s) \Delta s .
\end{aligned}
$$

This completes the proof.

Lemma 3.6 If $g \in \operatorname{DAP}^{\varepsilon}(\mathbb{T} \times \mathbb{X}, \mathbb{X})$ and $\alpha \in \operatorname{DAP}^{\varepsilon}(\mathbb{T}, \mathbb{X})$, then $G(t):=g(\cdot, \alpha(\cdot)) \in$ $\operatorname{DAP}^{\varepsilon}(\mathbb{T}, \mathbb{X})$.

Proof Let $T=\left\{t_{k_{i}, \varepsilon}\right\} \subset \mathfrak{B}^{\varepsilon}, \tilde{\phi}=(g(t, x), T) \in \mathrm{DAP}^{\varepsilon}(\mathbb{T} \times \mathbb{X}, \mathbb{X}) \times \mathfrak{B}^{\varepsilon}$, and from every sequence $\left\{s_{n}\right\}_{n=1}^{\infty} \subset \Pi_{\varepsilon}$, we can extract a subsequence $\left\{\tau_{n}\right\}_{n=1}^{\infty}$ and a sequence $\left\{A_{-\tau_{n}}\right\}$ such that the limit set $\mathbb{T}_{0}$ of $\left\{\mathbb{T}^{-\tau_{n}} \backslash A_{-\tau_{n}}\right\}$ exists and

$$
\tilde{\phi}^{*}:=\left(g^{*}(t, x), T^{*}\right)=\lim _{n \rightarrow \infty} \theta_{\tau_{n}} \tilde{\phi}=\lim _{n \rightarrow \infty}\left(g\left(t+\tau_{n}, x\right), T^{-\tau_{n}}\right),
$$

uniformly exists on $\operatorname{PC}_{r d}^{\varepsilon}\left(\mathbb{T}_{0} \times \mathbb{X}, \mathbb{X}\right) \times \mathfrak{B}^{\varepsilon}$. Since $\alpha \in \operatorname{DAP}^{\varepsilon}(\mathbb{T}, \mathbb{X})$, we can extract $\left\{\tau_{n}^{\prime}\right\} \subset\left\{\tau_{n}\right\}$ such that

$$
\begin{aligned}
\lim _{n \rightarrow \infty} \theta_{\tau_{n}^{\prime}} \tilde{\phi} & =\lim _{n \rightarrow \infty}\left(g\left(t+\tau_{n}^{\prime}, \alpha\left(t+\tau_{n}^{\prime}\right)\right), T^{-\tau_{n}^{\prime}}\right) \\
& =\lim _{n \rightarrow \infty}\left(g\left(t+\tau_{n}^{\prime}, \alpha^{*}(t)\right), T^{-\tau_{n}^{\prime}}\right)=\left(g^{*}\left(t, \alpha^{*}(t)\right), T^{*}\right) .
\end{aligned}
$$

Hence, $G \in \operatorname{DAP}^{\varepsilon}(\mathbb{T}, \mathbb{X})$. This completes the proof. 
Theorem 3.4 Let $f=g+\phi \in \operatorname{WPDAP}^{\varepsilon}(\mathbb{T} \times \mathbb{X}, \tilde{\rho})$, where $g \in \operatorname{DAP}^{\varepsilon}(\mathbb{T} \times \mathbb{X}, \mathbb{X})$, $\phi \in$ $\operatorname{WPDAP}_{0}^{\varepsilon}(\mathbb{T} \times \mathbb{X}, \tilde{\rho}), \tilde{\rho} \in U_{\infty}^{\varepsilon}$ and the following conditions hold:

(i) $\left\{f(t, x): t \in \mathcal{R}_{\mathbb{T}}(\tau, \varepsilon), x \in K\right\}$ is bounded for every bounded subset $K \subseteq \Omega$.

(ii) $f(t, \cdot), g(t, \cdot)$ are uniformly continuous in each bounded subset of $\Omega$ uniformly in $t \in \mathcal{R}_{\mathbb{T}}(\tau, \varepsilon)$.

Then, $f(\cdot, h(\cdot)) \in \operatorname{WPDAP}^{\varepsilon}(\mathbb{T}, \tilde{\rho})$ if $h \in \operatorname{WPDAP}^{\varepsilon}(\mathbb{T}, \tilde{\rho})$ and $h\left(\mathcal{R}_{\mathbb{T}}(\tau, \varepsilon)\right) \subset \Omega$.

Proof For any $\varepsilon_{1}>0$, we have $f=g+\phi$, where $g \in \operatorname{DAP}^{\varepsilon_{1}}(\mathbb{T} \times \mathbb{X}, \mathbb{X})$ and $\phi \in \operatorname{WPDAP}_{0}^{\varepsilon_{1}}(\mathbb{T} \times$ $\mathbb{X}, \tilde{\rho})$ and $h=\phi_{1}+\phi_{2}$, where $\phi_{1} \in \operatorname{DAP}^{\varepsilon_{1}}(\mathbb{T}, \mathbb{X})$ and $\phi_{2} \in \operatorname{WPDAP}_{0}^{\varepsilon_{1}}(\mathbb{T}, \tilde{\rho})$. Hence, the function $f(\cdot, h(\cdot))$ can be decomposed as

$$
\begin{aligned}
f(\cdot, h(\cdot)) & =g\left(\cdot, \phi_{1}(\cdot)\right)+f(\cdot, h(\cdot))-g\left(\cdot, \phi_{1}(\cdot)\right) \\
& =g\left(\cdot, \phi_{1}(\cdot)\right)+f(\cdot, h(\cdot))-f\left(\cdot, \phi_{1}(\cdot)\right)+\phi\left(\cdot, \phi_{1}(\cdot)\right) .
\end{aligned}
$$

By Lemma 3.6, $g\left(\cdot, \phi_{1}(\cdot)\right) \in \operatorname{DAP}^{\varepsilon_{1}}(\mathbb{T}, \mathbb{X})$. Now, consider the function

$$
\Psi(\cdot):=f(\cdot, h(\cdot))-f\left(\cdot, \phi_{1}(\cdot)\right)
$$

Clearly, $\Psi \in \mathrm{BPC}_{r d}^{\varepsilon_{1}}(\mathbb{T}, \mathbb{X})$. For $\Psi$ to be in $\operatorname{WPDAP}_{0}^{\varepsilon_{1}}(\mathbb{T}, \tilde{\rho})$, for any $\varepsilon_{2}>\varepsilon_{1}>0$, it is sufficient to show that

$$
\lim _{D_{r} \rightarrow \infty} \frac{1}{m\left(r_{1}, r_{2}, \tilde{\rho}\right)} \int_{M_{r_{1}, r_{2}, \varepsilon_{2}}(\Psi)} \tilde{\rho}(t) \Delta t=0 .
$$

Let $K$ be a bounded subset of $\Omega$ such that $\phi\left(\mathcal{R}_{\mathbb{T}}\left(\tau, \varepsilon_{1}\right)\right) \subseteq K, \phi_{1}\left(\mathcal{R}_{\mathbb{T}}\left(\tau, \varepsilon_{1}\right)\right) \subseteq K$. From (ii), $f(t, \cdot)$ is uniformly continuous in $\phi_{1}\left(\mathcal{R}_{\mathbb{T}}\left(\tau, \varepsilon_{1}\right)\right)$ uniformly in $t \in \mathcal{R}_{\mathbb{T}}\left(\tau, \varepsilon_{1}\right)$. Hence, for a given $\varepsilon_{2}>\varepsilon_{1}>0$, there exists $\delta_{\varepsilon_{2}}>0$ such that $y_{1}, y_{2} \in K$ and $\left\|y_{1}-y_{2}\right\|<\delta_{\varepsilon_{2}}$ implies that

$$
\left\|f\left(t, y_{1}\right)-f\left(t, y_{2}\right)\right\|<\varepsilon_{2}, \quad t \in \mathcal{R}_{\mathbb{T}}\left(\tau, \varepsilon_{1}\right)
$$

Thus, for each $t \in \mathcal{R}_{\mathbb{T}}\left(\tau, \varepsilon_{1}\right),\left\|\phi_{2}(t)\right\|<\delta_{\varepsilon_{2}}$ implies that uniformly in $t \in \mathcal{R}_{\mathbb{T}}\left(\tau, \varepsilon_{1}\right)$,

$$
\left\|f(t, h(t))-f\left(t, \phi_{1}(t)\right)\right\|<\varepsilon_{2}
$$

where $\phi_{2}(t)=h(t)-\phi_{1}(t)$. For $r_{1}, r_{2} \in \mathcal{R}_{\mathbb{T}}\left(\tau, \varepsilon_{1}\right), r_{2}>r_{1}$, let $M_{r_{1}, r_{2}, \delta_{\varepsilon_{2}}}\left(\phi_{2}\right)=\left\{t \in\left[r_{1}\right.\right.$, $\left.\left.r_{2}\right]_{\mathcal{R}_{\mathbb{T}}\left(\tau, \varepsilon_{1}\right)}:\left\|\phi_{2}\right\| \geq \delta_{\varepsilon_{2}}\right\}$, and we obtain

$$
\begin{aligned}
& \frac{1}{m\left(r_{1}, r_{2}, \tilde{\rho}\right)} \int_{M_{r_{1}, r_{2}, \varepsilon_{2}(\Psi(t))}} \tilde{\rho}(t) \Delta t \\
& \quad=\frac{1}{m\left(r_{1}, r_{2}, \tilde{\rho}\right)} \int_{M_{r_{1}, r_{2}, \varepsilon_{2}}\left(f(t, h(t))-f\left(t, \phi_{1}(t)\right)\right)} \tilde{\rho}(t) \Delta t \\
& \quad \leq \frac{1}{m\left(r_{1}, r_{2}, \tilde{\rho}\right)} \int_{M_{r_{1}, r_{2}, \delta_{2}}\left(h(t)-\phi_{1}(t)\right)} \tilde{\rho}(t) \Delta t \\
& \quad=\frac{1}{m\left(r_{1}, r_{2}, \tilde{\rho}\right)} \int_{M_{r_{1}, r_{2}, \delta_{\varepsilon_{2}}}\left(\phi_{2}(t)\right)} \tilde{\rho}(t) \Delta t .
\end{aligned}
$$


Now, since $\phi_{2} \in \operatorname{WPDAP}_{0}^{\varepsilon_{1}}(\mathbb{T}, \tilde{\rho})$, Lemma 3.1 yields that

$$
\lim _{D_{r} \rightarrow \infty} \frac{1}{m\left(r_{1}, r_{2}, \tilde{\rho}\right)} \int_{M_{r_{1}, r_{2}, \varepsilon_{2}}\left(\phi_{2}(t)\right)} \tilde{\rho}(t) \Delta t=0,
$$

which confirms that $\Psi \in \operatorname{WPDAP}_{0}^{\varepsilon_{1}}(\mathbb{T}, \tilde{\rho})$.

Finally, we will show that $\phi\left(\cdot, \phi_{1}(\cdot)\right) \in \operatorname{WPDAP}_{0}^{\varepsilon_{1}}(\mathbb{T}, \tilde{\rho})$. Note that $f=g+\phi$ and $g(t, \cdot)$ is uniformly continuous in $\phi_{1}\left(\mathcal{R}_{\mathbb{T}}\left(\tau, \varepsilon_{1}\right)\right)$ uniformly in $t \in \mathcal{R}_{\mathbb{T}}\left(\tau, \varepsilon_{1}\right)$. By assumption (ii), $f(t, \cdot)$ is uniformly continuous in $\phi_{1}\left(\mathcal{R}_{\mathbb{T}}\left(\tau, \varepsilon_{1}\right)\right)$ uniformly in $t \in \mathcal{R}_{\mathbb{T}}\left(\tau, \varepsilon_{1}\right)$, so is $\phi$. Since $\phi_{1}\left(\mathcal{R}_{\mathbb{T}}\left(\tau, \varepsilon_{1}\right)\right)$ is relatively compact in $\mathbb{X}$, for $\frac{\varepsilon_{2}}{2}>\varepsilon_{1}>0$, there exists $\delta_{\varepsilon_{2}}>0$ such that $\phi_{1}\left(\mathcal{R}_{\mathbb{T}}\left(\tau, \varepsilon_{1}\right)\right) \subset \bigcup_{k=1}^{m} B_{k}^{\varepsilon_{2}}$, where $B_{k}^{\varepsilon_{2}}=\left\{x \in \mathbb{X}:\left\|x-x_{k}\right\|<\delta_{\varepsilon_{2}}\right\}$ for some $x_{k} \in \phi_{1}\left(\mathcal{R}_{\mathbb{T}}\left(\tau, \varepsilon_{1}\right)\right)$, and

$$
\left\|\phi\left(t, \phi_{1}(t)\right)-\phi\left(t, x_{k}\right)\right\|<\frac{\varepsilon_{2}}{2}, \quad \phi_{1}(t) \in B_{k}^{\varepsilon_{2}}, t \in \mathcal{R}_{\mathbb{T}}\left(\tau, \varepsilon_{1}\right) .
$$

It is easy to see that the set $U_{k}^{\varepsilon_{2}}:=\left\{t \in \mathcal{R}_{\mathbb{T}}\left(\tau, \varepsilon_{1}\right): \phi_{1}(t) \in B_{k}^{\varepsilon_{2}}\right\}$ is open and for any $r_{1}, r_{2} \in$ $\mathcal{R}_{\mathbb{T}}\left(\tau, \varepsilon_{1}\right)$, we have $\left[r_{1}, r_{2}\right]_{\mathcal{R}_{\mathbb{T}}\left(\tau, \varepsilon_{1}\right)} \subseteq \bigcup_{k=1}^{m} U_{k}^{\varepsilon_{2}}$. We define $V_{1}^{\varepsilon_{2}}=U_{1}^{\varepsilon_{2}}, V_{k}^{\varepsilon_{2}}=U_{k}^{\varepsilon_{2}} \backslash \bigcup_{i=1}^{k-1} U_{i}^{\varepsilon_{2}}$, $2 \leq k \leq m$. Then, it is clear that $V_{i}^{\varepsilon_{2}} \cap V_{j}^{\varepsilon_{2}} \neq \emptyset$ if $i \neq j, 1 \leq i, j \leq m$. Thus, we have

$$
\begin{aligned}
& \left\{t \in\left[r_{1}, r_{2}\right]_{\mathcal{R}_{\mathbb{T}}\left(\tau, \varepsilon_{1}\right)}:\left\|\phi\left(t, \phi_{1}(t)\right)\right\| \geq \frac{\varepsilon_{2}}{2}\right\} \\
& \subset \bigcup_{k=1}^{m}\left\{t \in V_{k}^{\varepsilon_{2}}:\left\|\phi\left(t, \phi_{1}(t)\right)-\phi\left(t, x_{k}\right)\right\|+\left\|\phi\left(t, x_{k}\right)\right\| \geq \varepsilon_{2}\right\} \\
& \subset \bigcup_{k=1}^{m}\left(\left\{t \in V_{k}^{\varepsilon_{2}}:\left\|\phi\left(t, \phi_{1}(t)\right)-\phi\left(t, x_{k}\right)\right\| \geq \frac{\varepsilon_{2}}{2}\right\}\right. \\
& \left.\quad \cup\left\{t \in V_{k}^{\varepsilon_{2}}:\left\|\phi\left(t, x_{k}\right)\right\| \geq \frac{\varepsilon_{2}}{2}\right\}\right) .
\end{aligned}
$$

Now, in view of (3.2), it follows that

$$
\left\{t \in V_{k}^{\varepsilon_{2}}:\left\|\phi\left(t, \phi_{1}(t)\right)-\phi\left(t, x_{k}\right)\right\| \geq \frac{\varepsilon_{2}}{2}\right\}=\emptyset, \quad k=1,2, \ldots, m .
$$

Thus, we obtain

$$
\frac{1}{m\left(r_{1}, r_{2}, \tilde{\rho}\right)} \int_{M_{r_{1}, r_{2}, \frac{\varepsilon_{2}}{2}}\left(\phi\left(t, \phi_{1}(t)\right)\right)} \tilde{\rho}(t) \Delta t \leq \sum_{k=1}^{m} \frac{1}{m\left(r_{1}, r_{2}, \tilde{\rho}\right)} \int_{M_{r_{1}, r_{2}, \frac{\varepsilon_{2}}{2}}\left(\phi\left(t, x_{k}\right)\right)} \tilde{\rho}(t) \Delta t .
$$

Since $\phi \in \operatorname{WPDAP}_{0}^{\varepsilon_{1}}(\mathbb{T} \times \mathbb{X}, \tilde{\rho})$ and

$$
\lim _{D_{r} \rightarrow \infty} \frac{1}{m\left(r_{1}, r_{2}, \tilde{\rho}\right)} \int_{M_{r_{1}, r_{2}, \frac{\varepsilon_{2}}{2}}\left(\phi\left(t, x_{k}\right)\right)} \tilde{\rho}(t) \Delta t=0
$$

from Lemma 3.1 it follows that

$$
\lim _{D_{r} \rightarrow \infty} \frac{1}{m\left(r_{1}, r_{2}, \tilde{\rho}\right)} \int_{M_{r_{1}, r_{2}, \frac{\varepsilon_{2}}{2}}\left(\phi\left(t, \phi_{1}(t)\right)\right)} \tilde{\rho}(t) \Delta t=0 .
$$

Hence, $\phi\left(\cdot, \phi_{1}(\cdot)\right) \in \operatorname{WPDAP}_{0}^{\varepsilon_{1}}(\mathbb{T}, \tilde{\rho})$. This completes the proof. 
From Theorem 3.4 the following corollary is immediate:

Corollary 3.2 Let $f=g+\phi \in \operatorname{WPDAP}^{\varepsilon}(\mathbb{T}, \tilde{\rho})$, where $\rho \in U_{\infty}^{\varepsilon}$. Assume that $f$ and $g$ are Lipschitz in $x \in \mathbb{X}$ uniformly in $t \in \mathcal{R}_{\mathbb{T}}(\tau, \varepsilon)$. Then, $f(\cdot, h(\cdot)) \in \operatorname{WPDAP}^{\varepsilon}(\mathbb{T}, \tilde{\rho})$ if $h \in$ $\operatorname{WPDAP}^{\varepsilon}(\mathbb{T}, \tilde{\rho})$.

Next, we prove the following two lemmas which are useful in establishing our main results.

Lemma 3.7 If $\varphi(t)$ is double-almost-periodic on ACCTS and for any $\varepsilon>0, \inf _{i} t_{k_{i}, \varepsilon}^{q}=\theta_{\varepsilon}>0$, then $\left\{\varphi\left(t_{k_{i}, \varepsilon}\right)\right\}$ is a double-almost-periodic sequence for $\left\{t_{k_{i}, \varepsilon}\right\} \subset \mathfrak{B}^{\varepsilon}$.

Proof For $\varepsilon_{1}>0$, we construct a sequence $\left\{t_{k_{i}, \varepsilon_{1}}^{\prime}\right\} \subset \mathcal{R}_{\mathbb{T}}\left(\tau, \varepsilon_{1}\right)$ satisfying the condition

$$
\begin{cases}t_{k_{i}, \varepsilon_{1}}^{\prime}=t_{k_{i}, \varepsilon_{1}}, & \text { if } t_{k_{i}, \varepsilon_{1}} \text { is a left-scattered point in } \mathcal{R}_{\mathbb{T}}\left(\tau, \varepsilon_{1}\right) \\ t_{k_{i}, \varepsilon_{1}}^{\prime}=t_{k_{i}, \varepsilon_{1}}-2 \varepsilon_{1}, & \text { if } t_{k_{i}, \varepsilon_{1}} \text { is a left-dense point in } \mathcal{R}_{\mathbb{T}}\left(\tau, \varepsilon_{1}\right)\end{cases}
$$

where $i \in \mathbb{Z}$. For $\varepsilon_{2}>\varepsilon_{1}>0$, we choose numbers $r \in \Pi_{\varepsilon_{1}}, q \in \mathbb{Z}$ such that $\|\varphi(t+r)-\varphi(t)\|<$ $\varepsilon_{2}$ and $\left|t_{k_{i}, \varepsilon_{1}}^{q}-r\right|<v, 0<v<\varepsilon_{2}$, for all $\left|t-t_{k_{i}, \varepsilon_{1}}^{\prime}\right|>\varepsilon_{2}, t \in \mathcal{R}_{\mathbb{T}}\left(\tau, \varepsilon_{1}\right), i \in \mathbb{Z}$. Since $-v<t_{k_{i+q}, \varepsilon_{1}}-$ $t_{k_{i}, \varepsilon_{1}}-r<v$ in view of (3.3), we find that $0<2 \varepsilon_{2}-v \leq t_{k_{i+q}, \varepsilon_{1}}-t_{k_{i}, \varepsilon_{1}}^{\prime}-r<2 \varepsilon_{2}+v<3 \varepsilon_{2}$. Thus, if $t^{\prime}, t^{\prime \prime}$ belong to the same interval of continuity with $\left|t^{\prime}-t^{\prime \prime}\right|<3 \varepsilon_{2}$ then $\left\|\varphi\left(t^{\prime}\right)-\varphi\left(t^{\prime \prime}\right)\right\|<$ $o\left(3 \varepsilon_{2}\right)$. Now, assuming that $2 o\left(3 \varepsilon_{2}\right)+\varepsilon_{2}<\varepsilon_{2}^{\prime}<\theta_{\varepsilon_{1}}$, we find

$$
\begin{aligned}
\left\|\varphi\left(t_{k_{i+q}, \varepsilon_{1}}\right)-\varphi\left(t_{k_{i}, \varepsilon_{1}}\right)\right\| \leq & \left\|\varphi\left(t_{k_{i+q}, \varepsilon_{1}}\right)-\varphi\left(t_{k_{i}, \varepsilon_{1}}^{\prime}+r\right)\right\|+\left\|\varphi\left(t_{k_{i}, \varepsilon_{1}}^{\prime}+r\right)-\varphi\left(t_{k_{i}, \varepsilon_{1}}^{\prime}\right)\right\| \\
& +\left\|\varphi\left(t_{k_{i}, \varepsilon_{1}}^{\prime}\right)-\varphi\left(t_{k_{i}, \varepsilon_{1}}\right)\right\|<2 o\left(3 \varepsilon_{2}\right)+\varepsilon_{2}<\varepsilon_{2}^{\prime} .
\end{aligned}
$$

This completes the proof.

Lemma 3.8 A necessary and sufficient condition for a bounded sequence $\left\{a_{n}\right\}$ to be in $\operatorname{WPDAP}_{0}^{\varepsilon}(\mathbb{Z}, \tilde{\rho})$ is that there exist a uniformly continuous function $f \in \operatorname{WPDAP}_{0}^{\varepsilon}(\mathbb{T}, \tilde{\rho})$ and a discretization partition $\left\{t_{n}\right\} \subset \mathcal{R}_{\mathbb{T}}(\tau, \varepsilon)$ such that $f\left(t_{n}\right)=a_{n}, n \in \mathbb{Z}, \tilde{\rho} \in U_{B}^{\varepsilon}$.

Proof Necessity. Let $r_{1}, r_{2} \in \mathcal{R}_{\mathbb{T}}(\tau, \varepsilon), r_{2}>r_{1}$, and we partition the interval $\left[r_{1}, r_{2}\right]_{\mathcal{R}_{\mathbb{T}}(\tau, \varepsilon)}$ as follows:

$$
r_{n_{1}}:=r_{1} \leq\left\lceil r_{1}\right\rceil:=n_{1}<r_{n_{1}+1}<\cdots<r_{n_{2}-1}<\left\lfloor r_{2}\right\rfloor:=n_{2} \leq r_{2}:=r_{n_{2}} .
$$

Denote $\xi=\max _{j}\left\{r_{j+1}-r_{j}\right\}$, and define a function

$$
f(t)=a_{j}+\left(t-r_{j}\right)\left(a_{j+1}-a_{j}\right), \quad t \in\left[r_{j}, r_{j+1}\right]_{\mathcal{R}_{\mathbb{T}}(\tau, \varepsilon)}, n_{1} \leq j \leq n_{2}, j \in \mathbb{Z} .
$$

It is obviously uniformly continuous on $\mathcal{R}_{\mathbb{T}}(\tau, \varepsilon)$. To show $f \in \operatorname{WPDAP}_{0}^{\varepsilon}(\mathbb{T}, \tilde{\rho})$ it suffice to note that

$$
\begin{aligned}
& \frac{1}{m\left(r_{1}, r_{2}, \tilde{\rho}\right)} \int_{r_{1}}^{r_{2}}\|f(s)\| \tilde{\rho}(s) \Delta s \\
& =\frac{1}{m\left(r_{1}, r_{2}, \tilde{\rho}\right)} \sum_{j=n_{1}}^{n_{2}-1} \int_{r_{j}}^{r_{j+1}}\left\|a_{j}+\left(s-r_{j}\right)\left(a_{j+1}-a_{j}\right)\right\| \tilde{\rho}(s) \Delta s
\end{aligned}
$$




$$
\begin{aligned}
& \leq \frac{1}{m\left(r_{1}, r_{2}, \tilde{\rho}\right)} \sum_{j=n_{1}}^{n_{2}-1}\left(\left\|a_{j}\right\| \overline{\tilde{\rho}}\left(t_{j}\right) \xi+\left\|a_{j+1}-a_{j}\right\| \int_{r_{j}}^{r_{j+1}}\left(s-r_{j}\right) \tilde{\rho}(s) \Delta s\right) \\
& \leq \frac{1}{m\left(r_{1}, r_{2}, \tilde{\rho}\right)} \sum_{j=n_{1}}^{n_{2}-1} \xi\left\|a_{j}\right\| \overline{\tilde{\rho}}\left(t_{j}\right)+\frac{\left(\left\|a_{n_{2}}\right\|+\left\|a_{n_{1}}\right\| \xi^{2}\right.}{m\left(r_{1}, r_{2}, \tilde{\rho}\right)} \bar{\rho} \\
& \leq \frac{1}{\sum_{t_{j} \in\left[r_{1}, r_{2}\right]_{\mathbb{R}(\tau, \varepsilon)}} \tilde{\rho}\left(t_{j}\right)} \sum_{j=n_{1}}^{n_{2}-1} \xi\left\|f\left(t_{j}\right)\right\| \overline{\tilde{\rho}}\left(t_{j}\right)+\frac{\left\|a_{n_{2}}\right\|+\left\|a_{n_{1}}\right\|}{m\left(r_{1}, r_{2}, \tilde{\rho}\right)} \xi^{2} \bar{\rho} \\
& =\frac{C_{2}}{\sum_{j=n_{1}}^{n_{2}-1} \overline{\tilde{\rho}}\left(t_{j}\right)} \sum_{j=n_{1}}^{n_{2}-1} \xi\left\|f\left(t_{j}\right)\right\| \overline{\tilde{\rho}}\left(t_{j}\right)+\frac{\left\|a_{n_{2}}\right\|+\left\|a_{n_{1}}\right\|}{m\left(r_{1}, r_{2}, \tilde{\rho}\right)} \xi^{2} \bar{\rho} \rightarrow 0, \quad \text { as } n_{2}-n_{1} \rightarrow+\infty,
\end{aligned}
$$

where $\overline{\tilde{\rho}}\left(t_{j}\right)=\sup \left\{\tilde{\rho}(t): t \in\left[r_{j}, r_{j+1}\right)_{\mathcal{R}_{\mathbb{T}}(\tau, \varepsilon)}\right\}$ and $\bar{\rho}=\sup _{t \in \mathcal{R}_{\mathbb{T}}(\tau, \varepsilon)} \tilde{\rho}(t)$.

Sufficiency. For any $t_{n_{1}}<t_{n_{2}}$, we partition the interval $\left[t_{n_{1}}, t_{n_{2}}\right]_{\mathcal{R}_{\mathbb{T}}\left(\tau, \varepsilon_{1}\right)}$ as follows:

$$
t_{n_{1}} \leq\left\lceil t_{n_{1}}\right\rceil:=n_{1}<t_{n_{1}+1}<\cdots<t_{n_{2}-1}<\left\lfloor t_{n_{2}}\right\rfloor:=n_{2} \leq t_{n_{2}} .
$$

Let $1>\varepsilon_{2}>\varepsilon_{1}>0$, there exists a $\delta_{\varepsilon_{2}}>0$ such that for $t \in\left(t_{j}-\delta_{\varepsilon_{2}}, t_{j}\right)_{\mathcal{R}_{\mathbb{T}}\left(\tau, \varepsilon_{1}\right)}, j \in \mathbb{Z}$ and $n_{1} \leq j \leq n_{2}$, we have

$$
\|f(t)\| \tilde{\rho}(t) \geq\left(1-\varepsilon_{2}\right)\left\|f\left(t_{j}\right)\right\| \tilde{\rho}\left(t_{j}\right), \quad n_{1} \leq j \leq n_{2} .
$$

Hence,

$$
\begin{aligned}
& \int_{t_{n_{1}}}^{t_{n_{2}}}\|f(t)\| \tilde{\rho}(t) \Delta t \\
& \quad \geq \sum_{j=n_{1}+1}^{n_{2}} \int_{t_{j-1}}^{t_{j}}\|f(t)\| \tilde{\rho}(t) \Delta t \geq \sum_{j=n_{1}+1}^{n_{2}} \int_{t_{j}-\delta_{\varepsilon_{2}}}^{t_{j}}\|f(t)\| \tilde{\rho}(t) \Delta t \\
& \quad \geq \sum_{j=n_{1}+1}^{n_{2}} \delta_{\varepsilon_{2}}\left(1-\varepsilon_{2}\right)\left\|f\left(t_{j}\right)\right\| \tilde{\rho}\left(t_{j}\right) \geq \delta_{\varepsilon_{2}}\left(1-\varepsilon_{2}\right) \sum_{j=n_{1}+1}^{n_{2}}\left\|f\left(t_{j}\right)\right\| \tilde{\rho}\left(t_{j}\right),
\end{aligned}
$$

which implies that

$$
\begin{aligned}
& \frac{1}{m\left(t_{n_{1}}, t_{n_{2}}, \tilde{\rho}\right)} \int_{t_{n_{1}}}^{t_{n_{2}}}\|f(t)\| \tilde{\rho}(t) \Delta t \\
& \geq \delta_{\varepsilon_{2}}\left(1-\varepsilon_{2}\right) \frac{1}{m\left(t_{n_{1}}, t_{n_{2}}, \tilde{\rho}\right)} \sum_{j=n_{1}+1}^{n_{2}}\left\|f\left(t_{j}\right)\right\| \underline{\tilde{\rho}}\left(t_{j}\right) \\
& \geq \delta_{\varepsilon_{2}}\left(1-\varepsilon_{2}\right) \frac{1}{\sum_{t_{j} \in\left[t_{n_{1}}, t_{n_{2}}\right]_{\mathbb{R}_{\mathbb{T}}\left(\tau, \varepsilon_{1}\right)}} \overline{\tilde{\rho}}\left(t_{j}\right)} \sum_{j=n_{1}+1}^{n_{2}}\left\|f\left(t_{j}\right)\right\| \underline{\tilde{\rho}}\left(t_{j}\right) \\
& \geq \delta_{\varepsilon_{2}}\left(1-\varepsilon_{2}\right) \frac{1}{C_{2} \sum_{t_{j} \in\left[t_{n_{1}}, t_{n_{2}}\right]_{\mathcal{R}_{\mathbb{T}}\left(\tau, \varepsilon_{1}\right)}} \frac{\tilde{\rho}\left(t_{j}\right)}{n_{j=n_{1}+1}}\left\|f\left(t_{j}\right)\right\| \underline{\tilde{\rho}}\left(t_{j}\right)}
\end{aligned}
$$

From (3.4) and $f \in \operatorname{WPDAP}_{0}^{\varepsilon_{1}}(\mathbb{T}, \tilde{\rho})$, it follows that $f\left(t_{n}\right)=a_{n} \in \operatorname{WPDAP}_{0}^{\varepsilon_{1}}(\mathbb{Z}, \tilde{\rho})$. This completes the proof. 
From Lemma 3.8, we have the following result directly:

Theorem 3.5 A necessary and sufficient condition for a bounded sequence $\left\{a_{n}\right\}$ to be in $\operatorname{WPDAP}^{\varepsilon}(\mathbb{Z}, \tilde{\rho})$ is that there exist a uniformly continuous function $f \in \operatorname{WPDAP}^{\varepsilon}(\mathbb{T}, \tilde{\rho})$ and a discretization partition $\left\{t_{n}\right\} \subset \mathcal{R}_{\mathbb{T}}(\tau, \varepsilon)$ such that $f\left(t_{n}\right)=a_{n}, n \in \mathbb{Z}, \tilde{\rho} \in U_{B}^{\varepsilon}$.

Theorem 3.6 Assume that $\rho \in U_{\infty}^{\varepsilon}$ and the sequence of vector-valued functions $\left\{I_{k}\right\}_{k \in \mathbb{Z}}$ is weighted pseudo-almost-periodic, i.e., for any $x \in \Omega,\left\{I_{k}(x), k \in \mathbb{Z}\right\}$ is weighted pseudoalmost-periodic sequence. Suppose that $\left\{I_{k}(x): k \in \mathbb{Z}, x \in K\right\}$ is bounded for every bounded subset $K \subseteq \Omega, I_{k}(x)$ is uniformly continuous in $x \in \Omega$ uniformly in $k \in \mathbb{Z}$. If $h \in$ $\operatorname{WPDAP}^{\varepsilon}(\mathbb{T}, \tilde{\rho}) \cap \operatorname{UPC}^{\varepsilon}(\mathbb{T}, \mathbb{X})$ such that $h\left(\mathcal{R}_{\mathbb{T}}(\tau, \varepsilon)\right) \subset \Omega$, then $I_{k}\left(h\left(t_{k_{i}, \varepsilon}\right)\right)$ is weighted pseudo-double-almost-periodic.

Proof Fix $h \in \operatorname{WPDAP}^{\varepsilon}(\mathbb{T}, \tilde{\rho}) \cap \mathrm{UPC}^{\varepsilon}(\mathbb{T}, \mathbb{X})$. First we will show that $h\left(t_{k_{i}, \varepsilon}\right)$ is weighted pseudo-double-almost-periodic. Since $h=\phi_{1}+\phi_{2}$, where $\phi_{1} \in \operatorname{DAP}^{\varepsilon}(\mathbb{T}, \mathbb{X})$ and $\phi_{2} \in$ $\operatorname{WPDAP}_{0}^{\varepsilon}(\mathbb{T}, \tilde{\rho})$, it follows from Lemma 3.7 that the sequence $\phi_{1}\left(t_{k_{i}, \varepsilon}\right)$ is double-almostperiodic. To show $h\left(t_{k_{i}, \varepsilon}\right)$ is weighted pseudo-double-almost-periodic, we need to show that $\phi_{2}\left(t_{k_{i}, \varepsilon}\right) \in \operatorname{WPDAP}_{0}^{\varepsilon}(\mathbb{Z}, \tilde{\rho})$. From the assumption, $h, \phi_{1} \in \mathrm{UPC}^{\varepsilon}(\mathbb{T}, \mathbb{X})$, so is $\phi_{2}$.

For any $t_{k_{n_{1}}}, t_{k_{n_{2}}} \in \mathfrak{B}^{\varepsilon_{1}}$, we partition the interval $\left[t_{k_{n_{1}}}, t_{k_{n_{2}}}\right]_{\mathcal{R}_{\mathbb{T}}\left(\tau, \varepsilon_{1}\right)}$, and we repeat the same proof process of Sufficiency for Lemma 3.8, so we have $\phi_{2}\left(t_{k_{i}, \varepsilon_{1}}\right) \in \operatorname{WPDAP}_{0}^{\varepsilon_{1}}(\mathbb{Z}, \tilde{\rho})$. Hence, $h\left(t_{k_{i}, \varepsilon_{1}}\right)$ is weighted pseudo-double-almost-periodic.

Next, we will show that $I_{k}\left(h\left(t_{k_{i}, \varepsilon}\right)\right)$ is weighted pseudo-double-almost-periodic. Let

$$
\begin{array}{ll}
I(t, x)=I_{n}(x)+\left(t-r_{n}\right)\left[I_{n+1}(x)-I_{n}(x)\right], & t \in\left[r_{n}, r_{n+1}\right]_{\mathcal{R}_{\mathbb{T}}(\tau, \varepsilon)}, k_{n_{1}} \leq n \leq k_{n_{2}}, n \in \mathbb{Z}, \\
\Phi_{0}(t)=h\left(t_{n}\right)+\left(t-r_{n}\right)\left[h\left(t_{n+1}\right)-h\left(t_{n}\right)\right], & t \in\left[r_{n}, r_{n+1}\right]_{\mathcal{R}_{\mathbb{T}}(\tau, \varepsilon)}, k_{n_{1}} \leq n \leq k_{n_{2}}, n \in \mathbb{Z},
\end{array}
$$

where $\xi$ is as in Lemma 3.8. Since $\left\{I_{n}\right\}$ is weighted pseudo-almost-periodic sequence and $\left\{h\left(t_{n}\right)\right\}$ is weighted pseudo-double-almost-periodic sequence, by Lemma 3.8 and Theorem 3.5, it follows that $I \in \operatorname{WPDAP}^{\varepsilon}(\mathbb{T} \times \Omega, \tilde{\rho}), \Phi_{0} \in \operatorname{WPDAP}^{\varepsilon}(\mathbb{T}, \tilde{\rho})$. For every $t \in \mathcal{R}_{\mathbb{T}}(\tau, \varepsilon)$, there exists a integer $k_{n_{1}} \leq n \leq k_{n_{2}}, n \in \mathbb{Z}$ such that $\left|t-r_{n}\right| \leq \xi$, and hence

$$
\begin{aligned}
\|I(t, x)\| & \leq\left\|I_{n}(x)\right\|+\left|t-r_{n}\right|\left[\left\|I_{n+1}(x)\right\|+\left\|I_{n}(x)\right\|\right] \\
& \leq(1+\xi)\left\|I_{n}(x)\right\|+\xi\left\|I_{n+1}(x)\right\| .
\end{aligned}
$$

Since $\left\{I_{n}(x): k_{n_{1}} \leq n \leq k_{n_{2}}, n \in \mathbb{Z}, x \in K\right\}$ is bounded for every bounded set $K \subseteq \Omega,\{I(t, x)$ : $\left.t \in \mathcal{R}_{\mathbb{T}}(\tau, \varepsilon), x \in K\right\}$ is bounded for every bounded set $K \subseteq \Omega$. For every $x_{1}, x_{2} \in \Omega$, we have

$$
\begin{aligned}
\left\|I\left(t, x_{1}\right)-I\left(t, x_{2}\right)\right\| \leq & \left\|I_{n}\left(x_{1}\right)-I_{n}\left(x_{2}\right)\right\|+\left|t-r_{n}\right|\left[\left\|I_{n+1}\left(x_{1}\right)-I_{n+1}\left(x_{2}\right)\right\|\right. \\
& \left.+\left\|I_{n}\left(x_{1}\right)-I_{n}\left(x_{2}\right)\right\|\right] \\
\leq & (1+\xi)\left\|I_{n}\left(x_{1}\right)-I_{n}\left(x_{2}\right)\right\|+\xi\left\|I_{n+1}\left(x_{1}\right)-I_{n+1}\left(x_{2}\right)\right\| .
\end{aligned}
$$

From the fact that $I_{k}(x)$ is uniformly continuous in $x \in \Omega$ uniformly in $k \in \mathbb{Z}$, it follows that $I(t, x)$ is uniformly continuous in $x \in \Omega$ uniformly in $t \in \mathcal{R}_{\mathbb{T}}(\tau, \varepsilon)$. Thus, by Theorem 3.4, $I\left(\cdot, \Phi_{0}(\cdot)\right) \in \operatorname{WPDAP}^{\varepsilon}(\mathbb{T}, \mathbb{X})$. Again, using Lemma 3.8 and Theorem 3.5, we 
find that $I\left(t_{k_{i}, \varepsilon}, \Phi_{0}\left(t_{k_{i}, \varepsilon}\right)\right)$ is a weighted pseudo-double-almost-periodic sequence, that is, $I_{k}\left(h\left(t_{k_{i}, \varepsilon}\right)\right)$ is weighted pseudo-double-almost-periodic. This completes the proof.

From Theorem 3.6, the following corollary follows:

Corollary 3.3 Assume that the sequence of vector-valued functions $\left\{I_{k}\right\}_{k \in \mathbb{Z}}$ is weighted pseudo-almost-periodic, $\rho \in U_{B}^{\varepsilon}$, if there is a number $L>0$ such that

$$
\left\|I_{k}(x)-I_{k}(y)\right\| \leq L\|x-y\|
$$

for all $x, y \in \Omega, k \in \mathbb{Z}$, if $h \in \operatorname{WPDAP}^{\varepsilon}(\mathbb{T}, \rho) \cap \mathrm{UPC}^{\varepsilon}(\mathbb{T}, \rho)$ such that $h\left(\mathcal{R}_{\mathbb{T}}(\tau, \varepsilon)\right) \subset \Omega$, then $I_{k}\left(h\left(t_{k_{i}, \varepsilon}\right)\right)$ is weighted pseudo-double-almost-periodic.

In the following, we show a criterion for a relatively compact set in $\mathrm{PC}_{r d}^{\varepsilon}(\mathbb{T}, \mathbb{X})$.

Let $\tilde{h}_{0}: \mathbb{T} \rightarrow \mathbb{R}$ be a continuous function such that $\tilde{h}_{0}(t) \geq 1$ for all $t \in \mathbb{T}$ and $\tilde{h}_{0}(t) \rightarrow \infty$ as $|t| \rightarrow \infty$. Then, the space

$$
\mathrm{PC}_{h_{0}}^{\varepsilon}(\mathbb{T}, \mathbb{X})=\left\{\phi \in \mathrm{PC}_{r d}^{\varepsilon}(\mathbb{T}, \mathbb{X}): \lim _{|t| \rightarrow \infty} \frac{\|\phi(t)\|}{h_{0}(t)}=0\right\}
$$

endowed with the norm $\|\phi\|_{h_{0}}^{\varepsilon}=\sup _{t \in \mathcal{R}_{\mathbb{T}}(\tau, \varepsilon)} \frac{\|\phi(t)\|}{h_{0}(t)}$ is a Banach space, where $h_{0}(t)=$ $\left.\tilde{h}_{0}\right|_{\mathcal{R}_{\mathbb{T}}(\tau, \varepsilon)}$.

Theorem 3.7 A set $\mathbb{B} \subseteq \mathrm{PC}_{h_{0}}^{\varepsilon}(\mathbb{T}, \mathbb{X})$ is relatively compact if and only if

(1) $\lim _{|t| \rightarrow \infty} \frac{\|\phi(t)\|}{h_{0}(t)}=0$ uniformly for $\phi \in \mathbb{B}$.

(2) $\mathbb{B}_{t}=\{\phi(t): \phi \in \mathbb{B}\}$ is relatively compact in $\mathbb{X}$ for every $t \in \mathcal{R}_{\mathbb{T}}(\tau, \varepsilon)$.

(3) The set $\mathbb{B}$ is equicontinuous on each interval $\left(t_{k_{i}, \varepsilon}, t_{k_{i+1}, \varepsilon}\right)_{\mathcal{R}_{\mathbb{T}}(\tau, \varepsilon)}, i \in \mathbb{Z}$.

Proof Let $\mathrm{PC}_{r d}^{0, \varepsilon}(\mathbb{T}, \mathbb{X})=\left\{\phi \in \mathrm{PC}_{r d}^{\varepsilon}(\mathbb{T}, \mathbb{X}): \lim _{|t| \rightarrow \infty}\|\phi(t)\|=0\right\}$. It is clear that $\mathrm{PC}_{r d}^{0, \varepsilon}(\mathbb{T}, \mathbb{X})$ is isometrically isomorphic with the space $\mathrm{PC}_{h_{0}}^{\varepsilon}(\mathbb{T}, \mathbb{X})$. In order to prove Lemma 3.7, we only need to show that $\mathbb{B}^{*} \subseteq \mathrm{PC}_{r d}^{0, \varepsilon}(\mathbb{T}, \mathbb{X})$ is a relatively compact set if and only if

(a) $\lim _{|t| \rightarrow \infty}\|f(t)\|=0$ uniformly for $f \in \mathbb{B}^{*}$.

(b) $\mathbb{B}_{t}^{*}=\left\{f(t): f \in \mathbb{B}^{*}\right\}$ is relatively compact in $\mathbb{X}$ for every $t \in \mathcal{R}_{\mathbb{T}}(\tau, \varepsilon)$.

(c) The set $\mathbb{B}^{*}$ is equicontinuous on each interval $\left(t_{k_{i}, \varepsilon}, t_{k_{i+1}, \varepsilon}\right)_{\mathcal{R}_{\mathbb{T}}(\tau, \varepsilon)}, i \in \mathbb{Z}$.

Sufficiency. By $(a)$, for any $\varepsilon_{2}>\varepsilon_{1}>0$, there exists $\delta_{1}^{\varepsilon_{2}}>0$ such that

$$
\|f(t)\|<\varepsilon_{2}, \quad|t|>\delta_{1}^{\varepsilon_{2}}, f \in \mathbb{B}^{*} .
$$

By $(c)$, for the above $\varepsilon_{2}$, there exists $\delta: 0<\delta_{\varepsilon_{2}}<\delta_{1}^{\varepsilon_{2}}$, such that $t^{\prime}, t^{\prime \prime} \in\left(t_{k_{i}, \varepsilon_{1}}, t_{k_{i+1}, \varepsilon_{1}}\right)_{\mathcal{R}_{\mathbb{T}}\left(\tau, \varepsilon_{1}\right)}$, $i \in \mathbb{Z},\left|t^{\prime}-t^{\prime \prime}\right|<\delta_{\varepsilon_{2}}$ implies

$$
\left\|f\left(t^{\prime}\right)-f\left(t^{\prime \prime}\right)\right\|<\varepsilon_{2}, \quad \forall f \in \mathbb{B}^{*} .
$$

For the interval $\left[-\delta_{1}^{\varepsilon_{2}}, \delta_{1}^{\varepsilon_{2}}\right]_{\mathcal{R}_{\mathbb{T}}\left(\tau, \varepsilon_{1}\right)}$, there exists a set

$$
S=\left\{s_{1}, s_{2}, \ldots, s_{q}\right\} \subset\left[-\delta_{1}^{\varepsilon_{2}}, \delta_{1}^{\varepsilon_{2}}\right]_{\mathcal{R}_{\mathbb{T}}\left(\tau, \varepsilon_{1}\right)}, \quad s_{j} \neq t_{k_{i}, \varepsilon_{1}}, j=1,2, \ldots, q
$$


such that $\left|t-s_{j}\right|<\delta_{\varepsilon_{2}}$ and

$$
\left\|f(t)-f\left(s_{j}\right)\right\|<\varepsilon_{2}, \quad j=1,2, \ldots, q, f \in \mathbb{B}^{*} .
$$

For any sequence $\left\{f_{k}: k \geq 1\right\} \subseteq \mathbb{B}^{*}$, by $(b)$, we can extract a subsequence that converges at each point $t \in \mathcal{R}_{\mathbb{T}}\left(\tau, \varepsilon_{1}\right)$. Since $S$ is finite, for the above $\varepsilon_{2}>\varepsilon_{1}>0$, there exists $n_{0} \in \mathbb{N}$ such that for all $m, n \geq n_{0}$,

$$
\left\|f_{m}(t)-f_{n}(t)\right\|<\varepsilon_{2}, \quad t \in S
$$

Hence, for $t \in\left[-\delta_{1}^{\varepsilon_{2}}, \delta_{1}^{\varepsilon_{2}}\right]_{\mathcal{R}_{\mathbb{T}}\left(\tau, \varepsilon_{1}\right)}$, by (3.6) and (3.7), it follows that

$$
\left\|f_{m}(t)-f_{n}(t)\right\| \leq\left\|f_{m}(t)-f_{m}\left(s_{j}\right)\right\|+\left\|f_{m}\left(s_{j}\right)-f_{n}\left(s_{j}\right)\right\|+\left\|f_{n}\left(s_{j}\right)-f_{n}(t)\right\|<3 \varepsilon_{2} .
$$

For $|t|>\delta_{1}^{\varepsilon_{2}}$, by (3.5), we have

$$
\left\|f_{m}(t)-f_{n}(t)\right\|<2 \varepsilon_{2}
$$

Thus, $\left\{f_{k}: k \geq 1\right\}$ is almost-uniformly convergent on $\mathcal{R}_{\mathbb{T}}\left(\tau, \varepsilon_{1}\right)$, and hence $\mathbb{B}^{*} \subseteq \mathrm{PC}_{r d}^{0, \varepsilon_{1}}(\mathbb{T}$, $\mathbb{X})$ is a relatively compact set.

Necessity. Since $\mathbb{B}^{*} \subseteq \mathrm{PC}_{r d}^{0, \varepsilon_{1}}(\mathbb{T}, \mathbb{X})$ is relatively compact, for any $\varepsilon_{2}>\varepsilon_{1}>0$, there exist a finite number of functions $f_{1}, f_{2}, \ldots, f_{m}$ of $\mathbb{B}^{*}$ such that

$$
\left\|f-f_{j}\right\|_{\varepsilon_{1}}<\varepsilon_{2}, \quad j=1,2, \ldots, m, f \in \mathbb{B}^{*} .
$$

This finite set of functions $f_{1}, f_{2}, \ldots, f_{m}$ is equicontinuous, that is, for the above $\varepsilon_{2}>\varepsilon_{1}>0$, there exists a number $\delta_{2}^{\varepsilon_{2}}>0$ such that for any $t^{\prime}, t^{\prime \prime} \in\left(t_{k_{i}, \varepsilon_{1}}, t_{k_{i+1}, \varepsilon_{1}}\right)_{\mathcal{R}_{\mathbb{T}}\left(\tau, \varepsilon_{1}\right)}, i \in \mathbb{Z},\left|t^{\prime}-t^{\prime \prime}\right|<$ $\delta_{2}^{\varepsilon_{2}}$ implies that $\left\|f_{j}\left(t^{\prime}\right)-f_{j}\left(t^{\prime \prime}\right)\right\|<\varepsilon_{2}$. Now, using (3.8), for any $f \in \mathbb{B}^{*}$, we have

$$
\left\|f\left(t^{\prime}\right)-f\left(t^{\prime \prime}\right)\right\| \leq\left\|f\left(t^{\prime}\right)-f_{j}\left(t^{\prime}\right)\right\|+\left\|f_{j}\left(t^{\prime}\right)-f_{j}\left(t^{\prime \prime}\right)\right\|+\left\|f_{j}\left(t^{\prime \prime}\right)-f\left(t^{\prime \prime}\right)\right\|<3 \varepsilon_{2},
$$

which shows $(c)$. Since $f_{j} \in \mathbb{B}^{*}$, for the above $\varepsilon_{2}>\varepsilon_{1}>0$, there exist numbers $v_{j}^{\varepsilon_{2}}>0$ such that

$$
\left\|f_{j}(t)\right\|<\varepsilon_{2}, \quad|t|>v_{j}^{\varepsilon_{2}}, j=1,2, \ldots, m .
$$

Let $\delta_{3}^{\varepsilon_{2}}=\max \left\{v_{1}^{\varepsilon_{2}}, \ldots, v_{m}^{\varepsilon_{2}}\right\}$. Then by (3.8) and (3.9), for any $f \in \mathbb{B}^{*}$, it follows that

$$
\|f(t)\| \leq\left\|f(t)-f_{j}(t)\right\|+\left\|f_{j}(t)\right\|<2 \varepsilon_{2}, \quad|t|>\delta_{3}^{\varepsilon_{2}}
$$

which shows (a). Since $\mathbb{B}^{*}$ is relatively compact, for any sequence $\left\{f_{k}: k \geq 1\right\} \subseteq \mathbb{B}^{*}$, there exists a subsequence that converges almost-uniformly on $\mathcal{R}_{\mathbb{T}}\left(\tau, \varepsilon_{1}\right)$. Fix $t \in \mathcal{R}_{\mathbb{T}}\left(\tau, \varepsilon_{1}\right)$, in the sequence $\left\{f_{k}(t): k \geq 1\right\} \subseteq \mathbb{X}$, there exists a convergent subsequence. Therefore, for fixed $t \in \mathcal{R}_{\mathbb{T}}\left(\tau, \varepsilon_{1}\right)$, the set $\left\{f(t): f \in \mathbb{B}^{*}\right\}$ is relatively compact, which shows $(b)$. This completes the proof. 
Remark 3.2 The applications of weighted piecewise double-almost-periodic functions on impulsive evolution equations were investigated in the literature [33]. Moreover, the first results of some biological dynamic models, economic dynamic models, and neural networks with double-almost-periodicity were established in Sect. 4 of [33] and also in [34].

\section{Conclusion}

This paper introduces the concept of ACCTS and establishes a type of functions with double-almost-periodicity. Then, by introducing the concept of almost-uniform convergence for piecewise functions on ACCTS and using measure theory on time scales, some composition theorems of WPDAP and the completeness of the function space are obtained. The basic results established in this paper can be applied to study weighted pseudodouble-almost-periodic solutions for impulsive dynamic equations or other types of mathematical dynamic models in the real world.

\section{Acknowledgements}

We express sincere thanks to all the reviewers for their insightful comments and valuable suggestions to improve this manuscript.

\section{Funding}

This work is supported by Youth Fund of NSFC (No. 11601470), Tian Yuan Fund of NSFC (No. 11526181), and Dong Lu Youth Excellent Teachers Development Program of Yunnan University (No. wX069051), IRTSTYN and Joint Key Project of Yunnan Provincial Science and Technology Department of Yunnan University (No. 2018FY001(-014)).

Availability of data and materials

Not applicable.

\section{Competing interests}

The authors declare that they have no competing interests.

Authors' contributions

All authors contributed equally to the manuscript and typed, read and approved the final manuscript.

\section{Author details}

${ }^{1}$ Department of Mathematics, Yunnan University, Kunming, Yunnan, China. ${ }^{2}$ Department of Mathematics, Texas A\&M University-Kingsville, Kingsville, USA. ${ }^{3}$ Florida Institute of Technology, Melbourne, USA. ${ }^{4}$ School of Mathematics, Statistics and Applied Mathematics, National University of Ireland, Galway, Ireland. ${ }^{5}$ Department of Mathematics, Bharathiar University, Coimbatore, India.

\section{Publisher's Note}

Springer Nature remains neutral with regard to jurisdictional claims in published maps and institutional affiliations.

Received: 22 May 2019 Accepted: 14 October 2019 Published online: 21 October 2019

\section{References}

1. Hilger, S.: Ein Maßkettenkalkül mit Anwendung auf Zentrumsmannigfaltigkeiten. PhD thesis, Universität Würzburg (1988)

2. Agarwal, R.P., Bohner, M.: Basic calculus on time scales and some of its applications. Results Math. 35, 3-22 (1999)

3. Agarwal, R.P., Bohner, M., O’Regan, D., Peterson, A.: Dynamic equations on time scales: a survey. J. Comput. Appl. Math. 141, 1-26 (2002)

4. Bohner, M., Peterson, A.: Dynamic Equations on Time Scales: An Introduction with Applications. Birkhäuser Boston, Boston (2001)

5. Guseinov, G.S.: Integration on time scales. J. Math. Anal. Appl. 285, 107-127 (2003)

6. Erbe, L., Peterson, A., Tisdell, C.C.: Existence of solutions to second-order BVPs on time scales. Appl. Anal. 84, 1069-1078 (2005)

7. Kéré, M., N'Guérékata, G.M.: Almost automorphic dynamic systems on time scales. Panam. Math. J. 28, 19-37 (2018)

8. Hilger, S.: Analysis on measure chains-a unified approach to continuous and discrete calculus. Results Math. 18, 18-56 (1990)

9. Lakshmikantham, V., Sivasudaram, S., Kaymakçalan, B.: Dynamical Systems on Measure Chains. Kluwer Academic, Boston (1996)

10. Atici, F.M., Biles, D.C.: Further development of stochastic calculus on time scales. Panam. Math. J. 25, 13-24 (2015)

11. Wang, C., Agarwal, R.P., O' Regan, D.: Periodicity, almost periodicity for time scales and related functions. Nonauton. Dyn. Syst. 3, 24-41 (2016) 
12. Bochner, S.: Beiträge zur Theorie der fastperiodische Funktionen. I: Funktionen einer Variablen. Math. Ann. 96 119-147 (1927)

13. Fink, A.M.: Almost Periodic Differential Equations. Springer, New York (1974)

14. Blot, J., Cieutat, P., Ezzinbi, K.: New approach for weighted pseudo-almost periodic functions under the light of measure theory, basic results and applications. Appl. Anal. 92, 493-526 (2013)

15. Ezzinbi, K., Hachimi, M.A.: Existence of positive almost periodic solutions of functional equations via Hilbert's protective metric. Nonlinear Anal., Theory Methods Appl. 26, 1169-1176 (1996)

16. Zhang, C.: Pseudo almost periodic solutions of some differential equations. J. Math. Anal. Appl. 181, 62-76 (1994)

17. Zhang, C.: Pseudo almost periodic solutions of some differential equations II. J. Math. Anal. Appl. 192, 543-561 (1995)

18. Cuevas, C., Sepúlveda, A., Soto, H.: Almost periodic and pseudo-almost periodic solutions to fractional differential and integro-differential equations. Appl. Math. Comput. 218, 1735-1745 (2011)

19. Chang, Y., Zhao, Z., Nieto, J.J.: Almost periodic and pseudo almost periodic mild solutions to a partial differential equation VIA fractional operators. Numer. Funct. Anal. Optim. 32, 1219-1238 (2011)

20. Ding, H., Nieto, J.J.: A new approach for positive almost periodic solutions to a class of Nicholson's blowflies model. J. Comput. Appl. Math. 253, 249-254 (2013)

21. Wang, C., Agarwal, R.P.: Almost periodic solution for a new type of neutral impulsive stochastic Lasota-Wazewska timescale model. Appl. Math. Lett. 70, 58-65 (2017)

22. Diagana, T:. Existence of weighted pseudo-almost periodic solutions to some classes of nonautonomous partial evolution equations. Nonlinear Anal., Theory Methods Appl. 74, 600-615 (2011)

23. Diagana, T.: Weighted pseudo almost periodic functions and applications. C. R. Math. 343, 643-646 (2006)

24. Blot, J., Cieutat, P.: Completeness of sums of subspaces of bounded functions and applications. Commun. Math. Anal. 19, 43-61 (2016)

25. Zheng, Z., Ding, H.: On completeness of the space of weighted pseudo almost automorphic functions. J. Funct. Anal. 268, 3211-3218 (2015)

26. Ding, H., Liang, J., Xiao, T.: Weighted pseudo almost automorphic functions and WPAA solutions to semilinear evolution equations. J. Math. Anal. Appl. 409, 409-427 (2014)

27. Wang, C., Agarwal, R.P.: Uniformly rd-piecewise almost periodic functions with applications to the analysis of impulsive $\Delta$-dynamic system on time scales. Appl. Math. Comput. 259, 271-292 (2015)

28. Wang, C., Agarwal, R.P.: Relatively dense sets, corrected uniformly almost periodic functions on time scales, and generalizations. Adv. Differ. Equ. 2015, 312 (2015)

29. Agarwal, R.P., O'Regan, D.: Some comments and notes on almost periodic functions and changing-periodic time scales. Electron. J. Math. Anal. Appl. 6, 125-136 (2018)

30. N'Guérékata, G.M., Milce, A., Mado, J.C.: Asymptotically almost automorphic functions of order $n$ and applications to dynamic equations on time scales. Nonlinear Stud. 23, 305-322 (2016)

31. Wang, C.: Almost periodic solutions of impulsive BAM neural networks with variable delays on time scales. Commun. Nonlinear Sci. Numer. Simul. 19, 2828-2842 (2014)

32. Wang, C., Agarwal, R.P.: Almost periodic dynamics for impulsive delay neural networks of a general type on almost periodic time scales. Commun. Nonlinear Sci. Numer. Simul. 36, 238-251 (2016)

33. Wang, C., Agarwal, R.P., O’ Regan, D.: Weighted piecewise pseudo double-almost periodic solution for impulsive evolution equations. J. Nonlinear Sci. Appl. 10, 3863-3886 (2017)

34. Wang, C., Sakthivel, R.: Double almost periodicity for high-order Hopfield neural networks with slight vibration in time variables. Neurocomputing 282, 1-15 (2018)

\section{Submit your manuscript to a SpringerOpen ${ }^{\circ}$ journal and benefit from:}

- Convenient online submission

- Rigorous peer review

- Open access: articles freely available online

- High visibility within the field

- Retaining the copyright to your article

Submit your next manuscript at $\boldsymbol{s p r i n g e r o p e n . c o m ~}$ 